\title{
Armut in Deutschland
}

\section{Ein Vergleich zwischen den beiden Haushaltspanelstudien SOEP und PASS}

\author{
Jonas Beste (iD) - Markus M. Grabka • Jan Goebel
}

Eingegangen: 3. August 2017 / Angenommen: 20. Februar 2018 / Online publiziert: 3. April 2018

(C) Der/die Autor(en) 2018. Dieser Artikel ist eine Open-Access-Publikation.

Zusammenfassung Die Ergebnisse von Armutsanalysen auf Basis von Befragungsdaten unterliegen statistischen Unsicherheiten und möglichen systematischen Verzerrungen, deren Ursachen sowohl in der Pre-Data-Collection-Phase (z. B. bei der Stichprobenziehung), der Data-Collection-Phase (Unit- bzw. Item-Non-Response), als auch in der Post-Data-Collection-Phase (Gewichtung, Datengenerierung) liegen können. Um diese studienspezifischen Einflüsse bewerten zu können, kann ein Vergleich der Ergebnisse auf Basis von mehreren Datenquellen hilfreich sein. In dieser Untersuchung werden die Einkommensverteilungen, mehrere Armutsmaße, die identifizierten Armutsrisikoquoten von Subpopulationen, Armutsfaktoren, die Betroffenheit von dauerhafter Armut sowie Auf- und Abstiege mit den beiden Haushaltspanelstudien Panel „Arbeitsmarkt und soziale Sicherung“ (PASS) und soziooekonomisches Panel (SOEP) berechnet und miteinander verglichen. Als zusätzliche Referenz nutzen wir Ergebnisse zu Armut basierend auf dem Mikrozensus. Ziel dieses Vorhabens ist es, die Aussagekraft der Ergebnisse von Armutsanalysen besser einschätzen zu können. Zwischen den beiden Studien können signifikante Unterschiede in den Armutsmaßen festgestellt werden, welche sich allerding teilweise über den Beobachtungszeitraum auflösen. Eine Annäherung der in PASS bestimmten Armut an die Werte des SOEP kann entweder durch einen Ausfallsprozess in den ersten Erhebungsjahren der PASS-Studie oder durch eine Verbesserung der Datenqualität der Einkommensinformation bedingt sein. Die Resultate einer multivariaten Analyse auf die Wahrscheinlichkeit ein Einkommen unterhalb der Armutsschwelle aufzuweisen weichen zwischen den beiden Studien kaum voneinander ab. In der be-

J. Beste $(\bowtie)$

Institut für Arbeitsmarkt- und Berufsforschung (IAB), Regensburger Str. 100, 90478 Nürnberg,

Deutschland

E-Mail: jonas.beste@iab.de

M. M. Grabka · J. Goebel

Deutsches Institut für Wirtschaftsforschung (DIW), Mohrenstr. 58, 10117 Berlin, Deutschland 
obachteten Armutsdynamik weisen die beiden Panelstudien neben großen Gemeinsamkeiten auch klare Unterschiede auf. Insgesamt zeigt sich ein in vielen Bereichen vergleichbares Bild von relativer materieller Armut in den beiden Haushaltspanelstudien, das jedoch in einigen Punkten voneinander abweicht. Hieraus lässt sich die Relevanz für die Analyse von Armut anhand mehrerer verschiedener Datenquellen ableiten.

Schlüsselwörter Einkommen · Einkommensverteilungen · Relative Armut · Armutsindikatoren · Haushaltspanelstudie

\title{
Poverty in Germany
}

A comparison of the household panel studies SOEP and PASS

\begin{abstract}
Results of poverty analyses based on survey data are subject to statistical uncertainties and could be affected by systematic bias. Potential error sources occur in the pre data collection phase (e.g. sampling), the data collection phase (e.g. unit non response or item non response) as well as in the post data collection phase (e.g. weighting or data generation). In order to evaluate study specific influences, a comparison of results based on multiple data sources can be informative. In this project we compare income distributions, poverty indicators, poverty rates of subpopulations, determinants of poverty, the persistence of poverty and the dynamic of poverty over time based on the two household panel studies German panel study "Labour Market and Social Security" (PASS) and the German socio-economic panel (SOEP). As an additional reference we use findings on poverty based on the German Microcensus. The aim of this project is to better assess the validity of the results of poverty analyses. We find significant differences in the poverty rates between the two panel studies, which fade over time. An approximation can be caused either by an attrition process in the first years of the PASS study or by an improvement in the data quality of income information. However, results of a multivariate analysis on the probability of having an income below the poverty threshold are hardly different between the two studies. Looking at the persistence and dynamic of poverty the two panel studies lead to very similar results. Overall, there is a comparable picture of relative material poverty resulting from the two household panel studies, which differs in some specific aspects. From this, the relevance of replication studies and the importance of using multiple data sources for a research question can be derived.
\end{abstract}

Keywords Income - Income distributions - Relative poverty · Poverty indicators · Household panel studies

\section{Einleitung}

Armut stellt ein gesellschaftlich und politisch relevantes Thema dar, welches in den letzten Jahren in der öffentlichen Diskussion immer weiter an Bedeutung gewonnen hat. Dieser Bedeutungsgewinn ist sicherlich nicht zuletzt auf einen Zuwachs von Armut in Deutschland in den letzten 20 Jahren zurückzuführen. Wie dem fünften 
Armuts- und Reichtumsbericht der Bundesregierung (Bundesministerium für Arbeit und Soziales 2017) zu entnehmen ist, betrifft relative Einkommensarmut einen nennenswerten Teil der deutschen Gesellschaft. Im politischen Diskurs werden unterschiedliche Kennziffern zur Beschreibung von Armut auf Basis verschiedener Datenquellen herangezogen. Diese Kennziffern sind das Produkt von Analysen im Rahmen der Armutsforschung und Armutsberichterstattung. Zur Analyse von Einkommensarmut werden Haushaltsstudien benötigt, die entsprechende Armutsindikatoren erfassen. Um zudem Aussagen über individuelle Verläufe und somit die Dynamik von Armut machen zu können, müssen diese zusätzlich als Längsschnittdaten vorliegen.

Kennziffern bilden in der Sozialberichterstattung einen festen Bestandteil, um gesellschaftlich relevante Veränderungen zu beschreiben. Bei der Bestimmung von Kennziffern für eine Grundgesamtheit kann unter bestimmten Voraussetzungen auf Informationen einer Stichprobe dieser Grundgesamtheit zurückgegriffen werden. Mittels inferenzstatistischer Methoden können so Rückschlüsse auf die Grundgesamtheit gemacht werden. Beim Stichprobenverfahren können jedoch unterschiedliche Fehlerquellen Einfluss auf die Ergebnisse nehmen. Zum einen kann der Sampling Frame von der gewünschten Zielpopulation abweichen. Dies kann dazu führen, dass nur auf einen Teil der gewünschten Zielpopulation hochrechnet wird. Man spricht hier von Coverage Error. Wenn zudem nicht alle Personen des Sampling Frames die (korrekte) Chance haben in das Sample gezogen zu werden, kann Sampling Bias die Ergebnisse verzerren. ${ }^{1}$ Auch Unit-Non-Response kann, falls die Ausprägungen der relevanten Merkmale der nicht befragten Personen von denen der befragten Personen abweichen, über Non-Response Error zu verzerrten Ergebnissen führen. Weitere Verzerrungen in Form von Adjustment Errors können durch Korrekturen (z.B. die Gewichtung) zustande kommen (Groves et al. 2009, S. 49 ff.). ${ }^{2}$

Üblicherweise werden Armutsanalysen auf Basis von Befragungsdaten mit freiwilliger Teilnahme durchgeführt. Diese weisen spezifische Eigenschaften auf, welche bei der Interpretation der Ergebnisse zu berücksichtigen sind. Dies betrifft u.a. die Teilnahmebereitschaft sowie auch die Interviewsituation, bei der vor allem der Erhebungsmodus von Bedeutung ist. Z.B. kann das Thema einer Befragung die Bereitschaft einer Person zur Teilnahme an der Befragung beeinflussen. Dies führt dann zu sinkenden Unit-Non-Response-Raten. Und mit der Zahl der Befragungswellen nimmt die Kooperation bei der Befragung zu. Dies äußert sich vor allem in sinkenden Item-Non-Response-Raten als auch in einem Rückgang von gerundeten Angaben (Frick und Grabka 2010). Zudem gilt, dass die Befragungsqualität generell höher ist, wenn ein Interviewer bei der Befragung anwesend ist (Leeuw und Collins 1997).

\footnotetext{
1 Der Sampling Bias ist nicht zu verwechseln mit dem Sampling Error, für den durch Berechnung von Konfidenzintervallen kontrolliert werden kann.

2 Neben Adjustement Errors, die durch die Gewichtung entstehen können, liegen weitere potentielle Fehlerquellen vor. Diese ergeben sich z. B. durch eine nicht angemessene Imputation von fehlenden Antwortangaben (Item-Non-Response, vgl. z. B. Frick und Grabka 2005, 2010) oder durch eine Korrektur von Partial Unit-Non-Response (Frick et al. 2012). Dieser liegt vor, wenn in einem sonst befragungsbereiten Haushalt eine Person vollständig die Teilnahme an der Befragung verweigert. Auch durch ein Editing vermeintlicher Ausreißer können zusätzliche Verzerrungen in der Stichprobe auftreten.
} 
Neben den oben bereits erwähnten Fehlern auf Seite der Stichprobenverfahren können bei der Erhebung von Informationen zudem Messfehler auftreten. Dabei stellt sich zunächst das Problem der Validität einer Messung, d.h. ob durch das Messinstrument tatsächlich das gemessen wird, was gemessen werden soll. Ein Messfehler auf Personenebene kann zudem durch spezifische Effekte, wie z. B. durch soziale Erwünschtheit, eintreten. Zusätzlich kann ein Processing Error, z. B. bei der Verkodung von offenen Angaben oder bei der Korrektur nicht plausibler Angaben, zu verzerrten Ergebnissen führen (Groves et al. 2009, S. 41 ff.).

Eine Möglichkeit den Ergebnissen mehr Sicherheit zuzuweisen ist es, eine Replikation mit unterschiedlichen Daten durchzuführen. Hierzu werden Ergebnisse auf Basis von mehreren verschiedenen Datensätzen, die Informationen über eine vergleichbare Grundgesamtheit zu einem vergleichbaren Zeitraum enthalten, gegenübergestellt. Die Replikation stellt die Wiederholbarkeit von Ergebnissen als eine Voraussetzung wissenschaftlichen Vorgehens in den Mittelpunkt und zielt darauf ab, durch die wiederholte Durchführung ähnlich angelegter Studien, die Zuverlässigkeit von Ergebnissen besser abschätzen zu können und die Grenzen der Aussagekraft der Ergebnisse einer Untersuchung zu identifizieren. Sie soll einen Hinweis darauf geben, inwieweit die Ergebnisse generalisierbar sind und ob die Ergebnisse nicht nur durch die studienspezifischen Bedingungen entstanden sind (King 1995).

Ein Vergleich der erfassten Armut zwischen zwei Haushaltspanelstudien kann einen Hinweis darauf geben, wie zuverlässig Armutsindikatoren auf Basis der ausgewählten Befragungsdaten sind. Dabei können Unterschiede auch auf Defizite in den Mikrodatenbasen hinweisen. Durch den regelmäßigen Abgleich mit anderen Datenquellen können potentielle Fehler so möglichst frühzeitig identifiziert und korrigiert werden.

Zwei Haushaltspanelstudien, die Armutsanalysen für Deutschland zulassen, sind das sozio-oekonomische Panel (SOEP) vom Deutschen Institut für Wirtschaftsforschung (DIW) und das Panel „Arbeitsmarkt und soziale Sicherung“ (PASS) vom Institut für Arbeitsmarkt- und Berufsforschung (IAB). Die beiden Studien weisen viele Gemeinsamkeiten, aber auch Unterschiede auf. Das SOEP wurde mit seinen derzeit 33 Erhebungswellen bereits regelmäßig für Armutsanalysen genutzt. Diese Studie deckt ein weites Themenspektrum ab, mit dem sich vielfältige sozial- und wirtschaftswissenschaftliche Fragestellungen untersuchen lassen. Das PASS stellt bislang zehn Wellen im Scientific-Use-File (SUF) bereit und eignet sich aufgrund des spezifischen Stichprobenaufbaus für Analysen im Niedrigeinkommensbereich, insbesondere für Untersuchungen zu Armutsübergängen. Auch die PASS-Studie ist thematisch breit aufgestellt, legt die Schwerpunkte allerdings auf Fragen in den Bereichen Erwerbsbeteiligung, soziale Teilhabe, Lebenslagen und Erfahrungen im Grundsicherungsleistungsbezug. Zwischen den beiden Studien ergeben sich zudem Unterschiede in der Erhebung von Armutsindikatoren und dem Aufbau der Stichproben. Die Ergebnisse von Armutsanalysen auf Basis dieser beiden Datenquellen lassen sich trotz der Unterschiede der beiden Studien gut miteinander vergleichen. Dies gilt besonders, da auf die Messung von Armut nach dem Ressourcenansatz anhand vergleichbarer Messinstrumente für eine vergleichbare Grundgesamtheit zu einem vergleichbaren Zeitraum zurückgegriffen werden kann. 
Ziel dieser Arbeit ist es, die Ergebnisse von Armutsanalysen auf Grundlage der beiden Haushaltspanelstudien PASS und SOEP systematisch miteinander zu vergleichen. Zunächst werden in Abschn. 2 Indikatoren der Armuts- und Einkommensforschung beschrieben. In Abschn. 3 werden anschließend die in den Analysen verwendeten Daten genauer vorgestellt. Um die Ergebnisse auf Basis der Befragungsdaten PASS und SOEP besser einordnen zu können, wird an ausgewählten Stellen der Mikrozensus als Validierungsdatensatz herangezogen. Aufgrund der hohen Fallzahl und der Teilnahmepflicht weist diese Datenquelle auch am unteren Rand der Einkommensverteilung viele Beobachtungen auf. Anschließend werden in Abschn. 4 die Ergebnisse der einzelnen Analysen vorgestellt. Betrachtet werden Einkommensverteilungen, mehrere Armutsmaße, die identifizierten Armutsrisikoquoten von Subpopulationen, Armutsfaktoren, die Betroffenheit von dauerhafter Armut sowie Aufund Abstiege. In Abschn. 5 folgt abschließend eine Einordnung der Befunde.

\section{Indikatoren zur Analyse von Einkommensverteilung und Armut}

Untersuchungen zur Armut in Deutschland ziehen häufig den indirekten Ressourcenansatz heran. Der Ressourcenansatz geht von den ökonomischen Mitteln aus, die eine potentielle Versorgungslage beschreiben. Unter Armut wird ein Mangel an Ressourcen verstanden. Das Konzept der Einkommensarmut beruht auf der Annahme, dass das Einkommen als zentrale Ressourcengröße maßgeblich für den Lebensstandard einer Person verantwortlich ist.

Zur Analyse von Einkommensverteilungen und Armut können verschiedene Maße herangezogen werden. Als gängige Maße für die Ungleichverteilung dienen die Lorenzkurve und der daraus abgeleitete Gini-Koeffizient. Die Lorenzkurve zeigt die statistische Verteilung anhand einer graphischen Darstellung. Dabei drückt die Fläche zwischen der perfekten Gleichverteilungsgerade und der beobachteten LorenzKurve den Grad der Ungleichverteilung aus. Je größer diese Fläche ist, desto größer auch die Ungleichheit. Der Gini-Koeffizient gibt diesen Anteil mit einer Kennziffer zwischen 0 (absolute Gleichverteilung) und 1 (absolute Ungleichverteilung) wieder. Dieser lässt sich über die Formel

$$
G=\frac{2 \sum_{i=1}^{n} i x_{i}}{n \sum_{i=1}^{n} x_{i}}-\frac{n+1}{n}
$$

berechnen, wobei $x_{i}$ das Einkommen der Person $i$ (bei nach dem Merkmal $x$ sortierten Daten) und $n$ die Anzahl der Personen in der Population ist.

Armutsanalysen gehen häufig von einer relativen Armutsgrenze aus. Dabei wird als arm klassifiziert, wer weniger als einen bestimmten Anteil des mittleren Einkommens aufweist. Das mittlere Einkommen wird dabei meist über den Median bestimmt, da dieser gegenüber extremen Werten am Rand der Verteilung robuster ist als das arithmetische Mittel. Neben der in Analysen hauptsächlich verwendeten 
Grenze bei $60 \%$ - hierbei spricht man von der Armutsrisikogrenze - kann zusätzlich auch eine $40 \%$ Grenze verwendet werden, um strenge Armut abzubilden. ${ }^{3}$

Ein einfaches Armutsmaß besteht darin, die Anzahl der Personen zu zählen, die die Armutsschwelle unterschreiten und diese der Gesamtbevölkerung gegenüberzustellen. Hieraus ergibt sich die Armutsquote. Hierbei wird jedoch nicht die Einkommensverteilung innerhalb der Armutspopulation berücksichtigt. Anhand des so genannten FGT-Maßes (Foster et al. 1984) kann weiter differenziert werden und es lassen sich Aussagen über die Tiefe der Armut treffen. Die generelle Formel für das FGT-Maß lautet:

$$
F G T(\alpha)=\frac{1}{N} \sum_{i=1}^{H}\left(\frac{z-y_{i}}{z}\right)^{\alpha}
$$

Durch den Parameter $\alpha$ wird bei den $H$ Personen in Armut der $N$ Personen in der Gesamtpopulation die Lücke zur Armutsschwelle gewichtet und tiefere Armut stärker ins Maß aufgenommen. Während bei der Armutsquote (FGT0: $\alpha=0)$ der Abstand des individuellen Einkommens $y_{i}$ zur Armutsschwelle $z$ unberücksichtigt bleibt, stellt die Armutslücke (FGT1: $\alpha=1$ ) ein Maß für den durchschnittlichen Einkommensabstand zur Überwindung der Armutsgrenze dar. Sie gibt somit Auskunft über das Ausmaß der Armut. Bei der Berechnung der Armutsintensität (FGT2: $\alpha=2$ ) werden in Armut lebende Personen umso stärker gewichtet, je weiter ihr Einkommen unterhalb der Armutsgrenze liegt.

Zur Beschreibung der zeitlichen Dimension von Armut kann das Ausmaß an permanenter Armut ausgegeben werden. Permanent arm sind Personen, die sich dauerhaft im Armutsbereich befinden. Demgegenüber stehen Personen, die nur temporär arm waren oder nie von Armut betroffen sind. Die Dynamik von Armut lässt sich durch Übergangsraten in Armut bzw. aus Armut heraus beschreiben. Diese Raten geben an, wie hoch der Anteil der Personen ist, die den Armutsbereich von einem Jahr zum nächsten verlassen bzw. in den Armutsbereich eintreten.

\section{Datenbasis: Sozio-oekonomische Panel (SOEP) und Panel „Arbeitsmarkt und soziale Sicherung“(PASS)}

Für die Analysen in dieser Untersuchung werden die beiden Haushaltspanelstudien SOEP und PASS verwendet. ${ }^{4}$ Ein Vergleich der erfassten Armut nach dem Ressourcenansatz zwischen diesen beiden Befragungsdaten bietet sich aus drei Gründen an.

\footnotetext{
$3 \mathrm{Zu}$ Vergleichszwecken werden zudem häufig weitere Anteilswerte des Median wie die 50\%-und die $70 \%$-Grenzen ausgegeben.

${ }^{4}$ Für Analysen zu Armut in Deutschland lassen sich verschiedene Datenquellen heranziehen. Neben der Einkommens- und Verbraucherstichprobe (EVS), einer amtlichen Statistik über die Lebensverhältnisse privater Haushalte in Deutschland, die auch Informationen über die Ausstattung mit Gebrauchsgütern sowie die Konsumausgaben privater Haushalte enthält (Statistisches Bundesamt 2013), steht z. B. auch die europäisch vergleichende Studie Statistics on Income and Living Conditions (EU-SILC) zur Verfügung. In dieser Panelerhebung werden Informationen zur Lebenssituation und den Arbeitsbedingungen der Bevölkerung in allen Mitgliedsstaaten der europäischen Union erfasst (Atkinson und Marlier 2010). Neben
} 
Erstens werden die zur Verfügung stehenden Ressourcen in beiden Studien nach einem vergleichbaren Konzept erhoben. Zweitens lassen die erhobenen Informationen Rückschlüsse auf eine vergleichbare Grundgesamtheit zu. Und drittens sind die Informationen für einen vergleichbaren Zeitraum erfasst.

In den folgenden Analysen werden die Ressourcen über das zur Verfügung stehende monatliche Haushaltsnettoeinkommen definiert. Die Ergebnisse beziehen sich auf Personen in Privathaushalten in Deutschland. Die Untersuchung deckt einen Zeitraum von 2007 bis 2013 ab.

Das SOEP ist eine vom DIW seit 1984 jährlich durchgeführte Wiederholungsbefragung. Die Stichprobe umfasst im Untersuchungszeitraum pro Erhebungsjahr ca. 11.000 Haushalte mit ungefähr 20.000 Befragungspersonen. Das SOEP stellt eine Omnibusbefragung dar, bei der unter anderem die Themenschwerpunkte Arbeit, soziale Netzwerke, Gesundheit, Wohnen und Bildung kontinuierlich erhoben werden. Hierdurch lässt sich eine große Bandbreite von wissenschaftlichen Fragen bearbeiten. Die Auswahl der Befragten erfolgt über eine echte, mehrfach geschichtete Zufallsstichprobe, die aus mehreren Teilstichproben besteht. Im hier ausgewählten Untersuchungszeitraum (Welle X bis BD) stehen zwölf verschiedene Teilstichproben zur Verfügung. ${ }^{5}$ Es wird ein Multi-Mode Verfahren angewendet, d. h. um die Teilnahmebereitschaft zu maximieren, wird angestrebt, den für den Befragten angenehmsten Interviewmode anzubieten. Dies ist typischerweise ein Face-to-Face Interview entweder in Papierform (Paper-and-Pencil Interview, PAPI) oder mit Hilfe eines Laptops (Computer Assisted Personal Interview, CAPI). ${ }^{6}$ Des Weiteren stellt das SOEP die verwendeten Fragebögen in unterschiedlichen Sprachfassungen zur Verfügung, um die Teilnahmebereitschaft von Personen mit Migrationshintergrund zu erhöhen. Ausführliche Personeninterviews werden mit Personen über 16 Jahren durchgeführt. Der Median für die Interviewdauer liegt bei einem Personeninterview bei 30 Minuten. Einen Überblick über die Studie bietet Wagner et al. (2007).

Neben dem SOEP wird für die folgenden Untersuchungen das PASS, ein vom IAB im Rahmen der SGB-II-Forschung erhobenes Haushaltspanel, verwendet. Das PASS ist eine seit Ende 2006/Anfang 2007 jährlich durchgeführte Panelstudie. Der inhaltliche Aufbau der Befragung eignet sich besonders für Analysen zu Determinanten des Zugangs in den bzw. Abgangs aus dem Leistungsbezug des Arbeitslosengelds II (ALG II) und erlaubt detaillierte Beschreibungen und Analysen der Lebenslagen von Leistungsempfängern und Personen in einkommensschwachen Haushalten. Zu-

\footnotetext{
wissenschaftsgetragenen Surveys zur Armutsforschung werden auch vom Statistischen Bundesamt unterschiedliche Datenquellen zur Verfügung gestellt.

5 Diese zwölf Teilstichproben werden in die Analyse mit aufgenommen: „A“ Deutsche West, „B“ Ausländer West, „C“ Deutsche Ost, „D“ Zuwanderer, „E“ bevölkerungsrepräsentative Auffrischungsstichprobe 1998, „F“ bevölkerungsrepräsentative Auffrischungsstichprobe 2000, „G“ Hohe Einkommen, „H“ bevölkerungsrepräsentative Auffrischungsstichprobe 2006, „I“ Incentivierung, „J“ bevölkerungsrepräsentative Auffrischungsstichprobe 2011, ,K“ bevölkerungsrepräsentative Auffrischungsstichprobe 2012, ,M“ Migration.

${ }^{6}$ Für langjährige Teilnehmer der SOEP-Befragung gibt es auch die Möglichkeit den Fragebogen selbst auszufüllen, dies wird von gut $41 \%$ der Befragten in Jahre 2010 in Anspruch genommen Die Erhebung fand ursprünglich nur als PAPI statt, wird aber seit dem Jahr 1998 zunehmend auch als CAPI durchgeführt. Proxy-Interviews werden grundsätzlich im SOEP nicht zu gelassen, auch telefonische Interviews liegen nur vereinzelt vor.
} 
dem werden Erfahrungen der Befragten mit den Jobcentern und detaillierte Informationen zur Qualität der Beschäftigung regelmäßig erhoben. Darüber hinaus sind u. a. die Themenbereiche Bildung, Erwerbstätigkeit, soziale Netzwerke, Gesundheit und Migration regelmäßig Bestandteil des Befragungsprogramms. Die Analysen werden mit den ersten sieben Wellen des PASS durchgeführt, die einen Zeitraum von 2006/20077 bis 2013 abdecken. Pro Erhebungswelle werden ungefähr zwischen 12.000 bis 19.000 Personen in 8000 bis 13.000 Haushalte befragt.

Das Erhebungsdesign der Studie besteht aus einer zweistufigen Zufallsstichprobe. Um Aussagen sowohl über die Population der ALG-II-Leistungsbezieher als auch über die Gesamtbevölkerung zuzulassen, startete das PASS mit zwei Teilstichproben. Für die erste Teilstichprobe wurden Bedarfsgemeinschaften aus Prozessdaten der Bundesagentur für Arbeit (BA) gezogen. Bei der zweiten Teilstichprobe handelt es sich um eine Bevölkerungsstichprobe mit überproportionaler Berücksichtigung einkommensschwacher Haushalte. Dies dient dem Ziel, auch die Einstiege in den Leistungsbezug mit einer ausreichend großen Fallzahl beobachten zu können. Um die Repräsentativität über die Zeit gewährleisten zu können, wird jedes Jahr eine Zugangsstichprobe von neuen Leistungsempfängern in die Erhebung mit aufgenommen. Diese enthält Personen, die im Juli des Vorjahres zum ersten Mal Leistungen nach dem SGB II erhalten haben. Somit setzt sich das PASS bis zur siebten Welle aus zehn Teilstichproben zusammen (vgl. Berg et al. 2014, S. 14 ff.). ${ }^{8}$

Es wird versucht, innerhalb der ausgewählten Haushalte alle Personen ab einem Alter ab 15 Jahren zu interviewen. Die Befragung findet wahlweise in CAPI oder CATI (Computer Assisted Telephone Interview) statt. Im PASS können die Befragten zwischen verschiedenen Sprachversionen wählen. Neben dem Interview in deutscher Sprache, steht der Fragebogen auch in türkischer, arabischer oder russischer Sprache zu Verfügung. ${ }^{9}$ Das Haushaltsinterview dauert rund 25 Minuten. Das Personeninterview hat eine Dauer von durchschnittlich 30 Minuten (Median). Es besteht die Möglichkeit, den Befragungsdaten Informationen aus den administrativen Daten der BA zuzuspielen. Einen kurzen Überblick über die Erhebung geben Bethmann et al. (2013) und Trappmann et al. (2013).

Bei beiden Stichproben handelt es sich um echte Zufallsstichproben, die sich aus mehreren Teilstichproben zusammensetzen. Das Auswahlverfahren ist in beiden Studien mehrstufig. Dabei wird die Grundgesamtheit in einem ersten Schritt in Primäreinheiten (primary sampling units) eingeteilt, aus denen wiederum in weiteren Schritten Folgeeinheiten gezogen werden (Schnell et al. 2011, S. 274). Wie bei allen freiwilligen Befragungen stellt sich auch beim SOEP und beim PASS das Problem der Befragungsbereitschaft. Die Selektion, die daraus entstehen kann, ist durch eine

\footnotetext{
7 In den ersten drei Wellen von PASS begann die Datenerhebungsphase (im CATI) im Dezember und endete im Juli/August des Folgejahres. Seit der vierten Welle startet die Erhebungsphase im Februar und dauert bis in den September.

8 Diese zehn Teilstichproben werden in die Analyse mit aufgenommen: Bevölkerungsstichprobe W1, Leistungsbezieherstichprobe W1, Zugangsstichprobe W2, Zugangsstichprobe W3, Zugangsstichprobe W4, Bestandsauffrischung der Bevölkerungsstichprobe W5, Bestandsauffrischung der Leistungsbezieherstichprobe W5, Zugangsstichprobe W5, Zugangsstichprobe W6, Zugangsstichprobe W7.

9 Die türkische Fragebogenversion wurde bis inkl. Welle 9 angeboten. Seit Welle 10 steht die arabische Fragebogenversion zur Verfügung.
} 
Tab. 1 Zuordnung der Wellen in PASS und SOEP zu den einzelnen Kalenderjahren

\begin{tabular}{llllllll}
\hline Daten & Jahr & & & & & & \\
& 2007 & 2008 & 2009 & 2010 & 2011 & 2012 & 2013 \\
\hline PASS & 1 & 2 & 3 & 4 & 5 & 6 & 7 \\
SOEP & $24(\mathrm{X})$ & $25(\mathrm{Y})$ & $26(\mathrm{Z})$ & $27(\mathrm{BA})$ & $28(\mathrm{BB})$ & $29(\mathrm{BC})$ & $30(\mathrm{BD})$ \\
\hline
\end{tabular}

entsprechende Gewichtung auszugleichen, wie dies sowohl beim SOEP als auch beim PASS geschieht. ${ }^{10}$ Für die Gewichtung werden im SOEP Randinformationen aus dem Mikrozensus herangezogen, die z. B. das Alter, das Geschlecht oder den Migrationsstatus der Befragten umfassen (Kroh 2010). Im PASS wird eine Kalibrierung an Eckwerte des Statistischen Bundesamts, an Eckwerte der Statistik der BA und an Eckwerte des Mikrozensus vorgenommen (Berg et al. 2014, S. $172 \mathrm{ff}$.). Zudem ergibt sich hier die Besonderheit, dass für die Ausfallsgewichtung auf Informationen aus den administrativen Daten der BA zurückgegriffen werden kann.

Ein wesentlicher Unterschied zwischen den beiden Längsschnittstudien liegt im Zeitpunkt der Studienbeginne. Da das SOEP bereits seit 1984 erhoben wird, nehmen einige Befragte im hier untersuchten Zeitraum schon seit fast 30 Jahren teil. Bei PASS wiederum wird auch die erste Erhebungswelle aus dem Jahr 2006/07 in die Analysen mit aufgenommen, wodurch für alle Personen immer auch die Erstbefragung enthalten ist (zur Qualität von Einkommensdaten in Panelumfragen siehe Frick et al. 2007). Da die Panelattrition vor allem zu Beginn einer Studie recht hoch ist, heißt dies, dass im hier verwendeten Beobachtungszeitraum beim PASS gegenüber dem SOEP mehr Befragte mit nur einer Erhebung enthalten sind. ${ }^{11}$ Zudem ist bei Panelbefragungen mit besonderen Effekten mit der Teilnahmedauer (z. B. Lerneffekten bei bestimmten Fragen) zu rechnen. Dies kann besonders bei einem Vergleich mit dem ersten Erhebungsjahr (2007) Einfluss auf die Ergebnisse haben.

Im PASS findet die Feldphase in der Regel von Januar/Februar bis August/ September statt, im SOEP vorwiegend von Februar bis Juni. Da die Feldphasen der einzelnen Wellen zwischen den beiden Studien nur gering voneinander abweichen, ist davon auszugehen, dass diese Unterschiede für einen Vergleich vernachlässigbar klein sind. Es wird zur besseren Darstellung im Folgenden jeder Erhebungswelle jeweils ein Kalenderjahr zugewiesen. Die Zuteilung ist in Tab. 1 zu sehen. Einen

\footnotetext{
${ }^{10}$ Zur korrekten Bestimmung der statistischen Unsicherheit gilt es bei der Gewichtung das Stichprobendesign zu berücksichtigen.

11 Beim PASS ist zwischen den Erhebungswellen 1 und 2 eine hohe Panelattrition zu beobachten. Im Übergang von der ersten zur zweiten Welle konnten knapp 43\% der in Welle 1 befragten Personen in Welle 2 nicht wiederbefragt werden. Ein Teil hiervon konnte jedoch für die dritte Welle wiedergewonnen werden. Die mögliche Selektivität dieser Ausfälle soll durch die Gewichtung möglichst ausgeglichen werden. Schnell et al. (2010) konnten durch Analysen mit Prozessdaten der BA für die erste Welle des PASS feststellen, dass es nur zu einer geringen Selektivität im Hinblick auf persönliches Einkommen oder den ALG2-Bezug durch die nichtteilnehmenden Personen kommt. In den darauffolgenden Wellen wird im PASS eine deutlich höhere Panelstabilität erreicht. Diese beträgt zwischen 69-75\%. Dabei ist bei der Leistungsempfängerstichprobe eine höhere Attrition feststellbar. Im SOEP wird dagegen über den gesamten Beobachtungszeitraum von 2007 bis 2013 eine relativ hohe Panelstabilität erreicht, die je nach Teilstichprobe zwischen $90 \%$ und $95 \%$ liegt. Aber auch bei dieser Panelbefragung werden beim Übergang von der ersten (1984) auf die zweite Welle (1985) geringere Stabilitätsquoten erzielt (Kroh 2012).
} 
Effekt auf die Datenqualität kann auch die Organisation der Feldphase (z.B. das Interviewertraining) haben. PASS wird vom Befragungsinstitut infas durchgeführt, während im SOEP alle Teilsamples von Kantar public erhoben werden. Informationen über den Einfluss des Erhebungsinstituts auf die Datenqualität liegen uns aber nicht vor. ${ }^{12}$

$\mathrm{Zu}$ Vergleichszwecken werden die Armutskennziffern auf Basis einer dritten Datenquelle herangezogen. Dabei handelt es sich um den Mikrozensus (MZ). Der MZ ist eine repräsentative Haushaltsbefragung der amtlichen Statistik in Deutschland. Rund 830.000 Personen in etwa 370.000 Haushalten werden stellvertretend für die gesamte Bevölkerung zu ihren Lebensbedingungen befragt. Dies entspricht $1 \%$ aller Haushalte in Deutschland. Das Erhebungskonzept wurde ab Januar 2005 auf eine kontinuierliche Erhebung während des ganzen Jahres umgestellt. Wegen der gesetzlichen Auskunftspflicht, die bei den meisten Fragen besteht, besitzt der MZ bei Armutsanalysen einen besonderen Vorteil gegenüber freiwilligen Erhebungen. Die üblicherweise Untererfassung von einkommensschwachen Haushalten bei freiwilligen Erhebungen entfällt dadurch. Trotz dieser Auskunftspflicht liegt auch beim MZ Item-Non-Response bei der Frage nach dem aktuellen Haushaltsnettoeinkommen vor. ${ }^{13}$ Auch kann vermutet werden, dass es durch verpflichtende Teilnahme vermehrt zu bewusst falschen Angaben kommt. Das Einkommen wird im MZ ausschließlich in Kategorien erhoben, wodurch Armutsanalysen nur eingeschränkt möglich sind. Der MZ als SUF ist aktuell bis zum Erhebungsjahr 2012 verfügbar. Da der MZ als rotierende Panelstichprobe angelegt ist, bei der die Haushalte vier Jahre in Folge befragt werden, können eingeschränkt auch Längsschnittanalysen durchgeführt werden. Trotz der ungenaueren Erhebung des Einkommens kann der MZ für Armutsanalysen verwendet werden (Gerhardt et al. 2009). Auch wenn der Mikrozensus nicht als verlässlichere Datenquelle für Armutsanalysen betrachtet werden kann, dienen die damit bestimmten Einkommens- und Armutsmaße auf Basis einer sehr hohen Fallzahl hier als Referenzgröße.

\subsection{Messungen von Einkommen in SOEP, PASS und MZ}

Zur Bestimmung der Einkommensarmut wird das bedarfsgewichtete Haushaltsnettoeinkommen (auch Äquivalenzeinkommen genannt) eines Haushalts herangezogen. Die Bedarfsgewichtung dient dazu, die Einkommenssituation zwischen Haushalten unterschiedlicher Zusammensetzung vergleichen zu können. Dabei werden den Personen im Haushalt je nach Alter unterschiedliche Bedarfsgewichte zugeteilt. Nach der modifizierten OECD-Skala wird der ersten erwachsenen Person im Haushalte ein Wert von 1 zugeteilt, jeder weiteren Person ab 14 Jahren ein Wert von 0,5 und jeder Person bis unter 14 Jahren ein Wert von 0,3. Durch die Gewichtung weiterer Haushaltmitglieder mit einem Wert kleiner eins wird angenommen, dass sich durch

\footnotetext{
${ }^{12}$ Hinweise auf den Einfluss unterschiedlicher Erhebungsinstitute auf die Datenqualität zeigen sich z. B. im Bereich der Wahlforschung (Blumenberg et al. 2013).

${ }^{13}$ Im MZ liegt der Item-Non-Response bei der Frage zum Haushaltsnettoeinkommen im Untersuchungszeitraum zwischen 7 bis $9 \%$. Die Auswertungen zur Einkommensarmut beziehen sich ausschließlich auf Personen in Privathaushalten mit gültigen Einkommensangaben.
} 
gemeinsames Wirtschaften Ersparnisse (so genannte Skaleneffekte) erzielen lassen. Grundlage für das bedarfsgewichtete Einkommen ist das einem Haushalt zur Verfügung stehende Nettoeinkommen. Das Haushaltsnettoeinkommen ist die Summe der persönlichen Nettoeinkommen aus sämtlichen Einkommensarten aller Haushaltsmitglieder (z. B. Lohn oder Gehalt, Unternehmereinkommen, Rente, Pension, öffentliche Unterstützungen, Einkommen aus Vermietung und Verpachtung, Kindergeld, Wohngeld, Transferleistungen für Unterkunft und Heizung, etc.). Dieses wird in beiden Studien in mehreren Varianten erfasst. Zum einen wird das zum Zeitpunkt der Befragung aktuelle monatliche Haushaltsnettoeinkommen ${ }^{14}$, also die regelmäßigen Einkünfte nach Abzug von Steuern und Sozialabgaben zuzüglich erhaltener Sozialtransfers, durch den Haushaltsvorstand direkt erfragt. Dabei handelt es sich um das sogenannte Screener-Einkommen (siehe Anhang). Zum anderen werden - im PASS für den letzten Monat, im SOEP zusätzlich auch für das zurückliegende Jahr - alle individuellen (Brutto-)Einkommen aller aktuell im Haushalt befragten Personen und deren gemeinsame Einkommen erhoben. Aus den aufsummierten Werten können, mit Hilfe einer Schätzung der Steuer- und Sozialabgaben und imputierter Werte für fehlende Angaben bzw. nicht befragungsbereiter Personen, die Jahresnettoeinkommen des Vorjahres bzw. Nettoeinkommen des Vormonats errechnet werden. Hierbei spricht man von Komponenten-Einkommen. Beim SOEP wird zudem der Mietwert selbstgenutzten Wohneigentums berücksichtigt sowie für Mieter mit subventionierter Miete auch der fiktive Einkommensvorteil gegenüber einer marktüblichen Miete erfasst (imputed rent) (Frick et al. 2010).

Es gilt die Einflüsse der beiden verschiedenen Arten der Einkommensmessung auf die Ergebnisse zu berücksichtigen. Beim direkt von einem Haushaltsmitglied erhobenen aktuellen Screener-Einkommen wird, vor allem mit zunehmender Haushaltsgröße, die Höhe des verfügbaren Einkommens eher unterschätzt, da bestimmte Einkommenskomponenten oder Einkommen von Haushaltsmitgliedern vergessen werden oder nicht bekannt sind (vgl. Groh-Samberg 2009, S. 127). In Goebel et al. (2009) werden Daten beider Einkommenskonzepte verwendet. In dieser Untersuchung wird darauf hingewiesen, dass es zwischen den beiden Konzepten aufgrund von Sonderzahlungen und Steuerrückzahlungen bei den Vorjahreseinkommen, als auch durch unterjährige Schwankungen beim Einkommensbezug z. B. aufgrund von saisonaler Arbeitslosigkeit, zu einer etwas höheren Ungleichheit und daher zu höheren Armutsquoten kommt. Es konnten aber abgesehen von diesen Niveaueffekten für beide Einkommenskonzepte dieselben Trends im zeitlichen Verlauf festgestellt werden.

Ein Vorteil des aktuellen Screener-Einkommens ist die Konsistenz von Haushaltsstruktur und Einkommen. Während beim Vorjahr als Bezugszeitraum eine Veränderung der Haushaltskonstellation möglich ist, wird beim aktuellen Monatseinkommen der gleiche zeitliche Bezugsrahmen von Soziodemografie und Einkommen verwendet. Auch dürfte es den meisten Personen (besonders bei Schwankungen im Einkommen) leichter fallen, valide Angaben zum aktuellen Monat zu machen als zum gesamten Vorjahr. Zudem erlaubt das Monatseinkommen eine bessere Vergleichbar-

14 Bei PASS wird nach dem Haushaltseinkommen im letzten Monat, im SOEP nach dem Haushaltseinkommen heute gefragt. Die Unterschiede dürften nur marginal sein. 
keit mit anderen Datenquellen wie z.B. dem MZ, der den gleichen Referenzzeitpunkt beim Haushaltseinkommen verwendet. Und auch die höhere Aktualität der Information spricht für diese Art der Einkommensmessung. ${ }^{15}$

Eine wichtige Frage bei der Analyse von Einkommen betrifft den Umgang mit fehlenden Angaben. Werden Personen in Haushalten mit fehlenden Angaben aus den Analysen ausgeschlossen (Case-Wise-Deletion), kann dies zu verzerrten Schätzern führen, falls der Ausfallprozess nicht einem reinen Zufallsprozess unterliegt (Missing completely at random, MCAR). Ein Vorteil des Screener-Einkommens gegenüber dem Komponenten-Einkommen ist, dass nur bei einer Variablen fehlende Werte auftreten und entsprechend nur eine Einkommenskomponente imputiert werden muss, während beim Komponenteneinkommen sowohl bei jeder Einzelkomponente als auch bei jeder zu befragenden Person Non-Response auftreten kann. ${ }^{16}$

Das Screener-Einkommen wird im SOEP auf Basis einer multiplen Imputation nach dem Chained-Equation Ansatz imputiert (Van Buuren und Groothuis-Oudshoorn 2000). ${ }^{17}$ Beim PASS wurde das Einkommen in der ersten Welle nur als Screener-Einkommen erhoben. In den folgenden Wellen wurden zusätzlich die einzelnen Einkommenskomponenten erfasst, imputierte Werte stehen jedoch zurzeit nicht zur Verfügung. Im Mikrozensus werden das persönliche Nettoeinkommen der einzelnen Haushaltsmitglieder und das Haushaltsnettoeinkommen im Monat vor der Befragung in 24 Einkommensklassen erhoben. Item-Non-Response liegt trotz des verpflichtenden Charakters des MZ vor und wird nicht durch Imputation korrigiert. ${ }^{18}$ Aufgrund dieser Erhebungsform bedarf es eines angemessenen Verfahrens zur Bestimmung der Armutsgrenze. Dabei wird den Haushalten nahe der Armutsschwelle eine Armutswahrscheinlichkeit zwischen 0 und 1 zugeordnet (für eine genaue Beschreibung des Verfahrens siehe Stauder und Hüning 2004 und Gerhardt et al. 2009). Eine wichtige Einschränkung des MZ betrifft die Verwendung von Proxyinterviews. Falls eine Person während der Feldphase nicht anzutreffen ist - vor allem im Falle von mobilen Personen wie junge Erwachsene, Pendler, etc. - werden im MZ auch Proxyinterviews eingesetzt, d.h. eine Referenzperson gibt stellvertretend Auskunft über die

15 Gemäß der Canberra Group und den Empfehlungen von EUROSTAT ist jedoch bei Einkommensverteilungsanalysen und Armutsanalysen ein Jahreseinkommen heranzuziehen.

16 Für das SOEP stehen Imputationen für die einzelnen Einkommenskomponenten in allen sieben relevanten Wellen bereit. Die Imputation basiert dabei auf der von Little und Su (1989) vorgeschlagenen Methode einer Kombination von längs- und querschnittbasierten Informationen der Befragten. Simulationsstudien z. B. von Watson und Starick (2011) deuten darauf hin, dass diese Imputationsmethode im Vergleich zu typischen Single-Imputationsverfahren überlegen ist. Liegen keine individuellen Längsschnittinformationen vor werden im SOEP im Regelfalle rein querschnittbasierte OLS-Regressionen herangezogen (Grabka und Frick 2003).

17 Der Anteil des Item-Non-Response beim Screener-Einkommen im SOEP beträgt knapp $7 \%$ im Jahre 2010. Der Anteil fehlender Antwortangaben bei den verschiedenen Einkommenskomponenten die im SOEP erfragt werden, schwankt zwischen gut $2 \%$ bei dem Betrag der von Unterhaltsvorschusskassen geleistet wird, über $10 \%$ beim Einkommen aus abhängiger Beschäftigung bis zu $18 \%$ bei Einkommen aus einer selbständigen Tätigkeit. Der Anteil des Partial Unit-Non-Response beträgt im SOEP knapp sieben Prozent (Frick et al. 2012).

18 Für Haushalte mit selbständigen Landwirten in der Haupttätigkeit wird im MZ das Haushaltseinkommen nicht erhoben (Boehle 2015, S. 9). Da für fehlende Werte keine Imputationen angeboten werden, bietet der MZ zwar eine sehr große Fallzahl, weist bei der Berechnung von Armutsquoten aber damit auch Nachteile auf. 
abwesende Person. Hierbei ist nicht auszuschließen, dass es zu einer Untererfassung der Erwerbs- und damit verbunden der Einkommenssituation des Haushalts kommen kann.

Bei den folgenden Analysen wird wegen der besseren Vergleichbarkeit das Screener-Einkommen verwendet, da nur dieses für alle drei Datenquellen vorliegt. Da für das PASS keine Imputationen bereit stehen, werden diese für die Analysen erstellt. Das Verfahren wird weiter unten im Abschnitt Datenaufbereitung beschrieben. Gegenüber dem komplexen Imputationsverfahren im SOEP handelt es sich bei den durchgeführten Imputationen um ein vergleichsweise simples Verfahren. Da der Anteil von Item-Non-Response verhältnismäßig klein ist ${ }^{19}$, wird die Bedeutung der Unterschiede in den Imputationsmethoden zwischen PASS und SOEP auf die folgenden Analysen als eher gering eingeschätzt.

\subsection{Datenaufbereitungsschritte}

Bevor die Armutsanalysen durchgeführt werden können, müssen die Datensätze vorbereitet werden. Hierfür gilt es, in den beiden Panelbefragungen spezifische Gegebenheiten zu beachten. Während im SOEP für das Screener-Einkommen imputierte Werte für fehlende Angaben zur Verfügung stehen, kann im PASS einem Großteil der Haushalte mit fehlenden Angaben durch die kategoriale Nachfrage ein Wert zugewiesen werden. Damit nicht alle Haushalte, die die gleiche Einkommenskategorie angegeben haben, einen identischen Wert zugewiesen bekommen (was u. a. zu Unschärfen bei der Bestimmung des Medians führen kann), werden Werte innerhalb des Wertebereiches der jeweiligen Kategorie vergeben. Die Verteilung orientiert sich an der gewichteten Verteilung der beobachteten Werte, die zwischen der jeweiligen Klassenober- und -untergrenze liegen. Für die restlichen Fälle wird eine regressionsbasierte Imputation durchgeführt. Hierbei wird für jede Erhebungswelle ein lineares Regressionsmodell mit den unabhängigen Variablen Wohnregion, Haushaltsgröße, Anzahl der erwachsenen Personen im Haushalt, Anzahl der erwerbstätigen Personen im Haushalt und Bezug von Arbeitslosengeld II im Haushalt und der zu erklärenden Variablen Haushaltsnettoeinkommen geschätzt. ${ }^{20}$ Anhand der Koeffizienten kann für die Haushalte ohne gültige Einkommensangabe ein Wert vorhergesagt werden. Diesen vorhergesagten Werten werden beobachtete Residuen zufällig aufaddiert, um die Varianz des Schätzers zu erhalten. Im SOEP liegen fünf multiple Imputationen für das Screener-Einkommen vor. Daher werden auch fünf multiple Imputationen für das Screener-Einkommen im PASS erstellt, indem jeweils ein unterschiedliches Residuum auf den vorgesagten Wert aufaddiert wird. Da für die fehlenden Werte beim Screener-Einkommen multiple Imputationen zum Einsatz kommen, werden die Punktschätzer als Durchschnitt über die Imputationen und die Standardfehler aus der Varianz innerhalb der Imputationen (Within-Imputation) sowie der Varianz

\footnotetext{
${ }_{19}$ Bei PASS liegen die Anteile der Personen ohne Angaben zur genauen Höhe des Einkommens in der ersten Welle bei $12 \%$, in den darauf folgenden Wellen zwischen 4 bis $7 \%$. Wenn die Angaben der kategorialen Einkommensabfrage berücksichtigt werden, sinken die Anteile auf $3 \%$ in der ersten Welle, $2 \%$ in den Wellen 2 bis 3 und $1 \%$ in den Wellen 4 bis 7.

${ }^{20}$ Das Adjusted-R-Quadrat für diese Regression beträgt zwischen 0,23 (in Welle 2) und 0,41 (in Welle 4).
} 
zwischen den Imputationen (Between-Imputation) berechnet (Krug 2010, S. 32f.). Dieses Vorgehen wird bei der Berechnung der Armutskennziffern angewendet. Bei den weiteren Berechnungen wird die jeweils erste Imputation verwendet.

Für den Vergleich der Armutspopulationen werden verschiedene Gruppen von Merkmalsträgern gebildet. Diese befinden sich zum einen auf der Haushaltsebene. So werden Haushaltstyp, Haushaltsgröße, Arbeitslosengeld-II-Bezug im Haushalt, Wohnregion und Wohnstatus verglichen. Zum anderen handelt es sich um Merkmale auf der Personenebene. Hier werden Bildung, Gesundheitszustand, Migration, Erwerbsstatus, berufliche Stellung und Familienstand verglichen. Die Analysen werden alle auf der Personenebene durchgeführt. Für die Merkmale auf der Haushaltsebene werden die Analysen für alle Personen in den Haushalten durchgeführt. Für die Merkmale auf Ebene der Personen werden die Analysen nur für Personen ab 18 Jahren erstellt. ${ }^{21}$

\section{Empirische Analysen}

\subsection{Einkommensverteilungen}

Für einen Vergleich mit den Einkommensangaben im MZ werden die Einkommensangaben aus SOEP und PASS in die beim MZ verwendeten Einkommensklassen eingeteilt. Dabei wird hier auf das Haushaltsnettoeinkommen vor Bedarfsgewichtung zurückgegriffen. Zudem wird auf die Verwendung von Imputationen verzichtet. Die kategorialen Angaben in PASS werden hier jedoch berücksichtigt. Hierdurch soll erkennbar werden, ob die Unterschiede bereits in den original erhobenen Screener-Einkommen ohne Korrektur durch Imputationen und Bedarfsgewichtung vorliegen. Abb. 1 gibt die Verteilung der Einkommen für die Jahre 2007 und 2012 wieder. Im Mittelpunkt stehen die Bereiche, bei denen stärkere Abweichungen in den Verteilungen zwischen SOEP und PASS beobachtet werden. Im Jahr 2007 liegen im Bereich bis $1100 €$ die Anteilswerte im MZ näher an denen im SOEP. Die Einkommensklasse von 1500 bis $1700 €$ weist im SOEP in 2007 um etwa zwei Prozentpunkte höhere Anteile auf als in den beiden anderen Studien. Dafür ist die Klasse von 1700 bis $2000 €$ im PASS etwas stärker besetzt. Im Jahr 2011 fallen die Unterschiede zwischen den drei Datenquellen geringer aus. Die Anteilswerte der unteren Einkommensklassen im PASS weichen nur noch geringfügig von denen im MZ ab. In einzelnen höheren Einkommensklassen liegen die Anteile bei PASS und SOEP (Klassen von 2000 bis 2600) über denen im MZ. Generell weist das PASS, aufgrund der Erhebung von Einkommensklassen bei der Nichtnennung der genauen Einkommenshöhe, nur einen vergleichsweise geringen Anteil von fehlenden Angaben auf.

\footnotetext{
${ }^{21}$ Der Grund hierfür ist, dass die vollständigen Personeninterviews, deren Informationen hier verwendet werden, im PASS ab 15 Jahren und im SOEP ab 17 Jahren durchgeführt werden.
} 


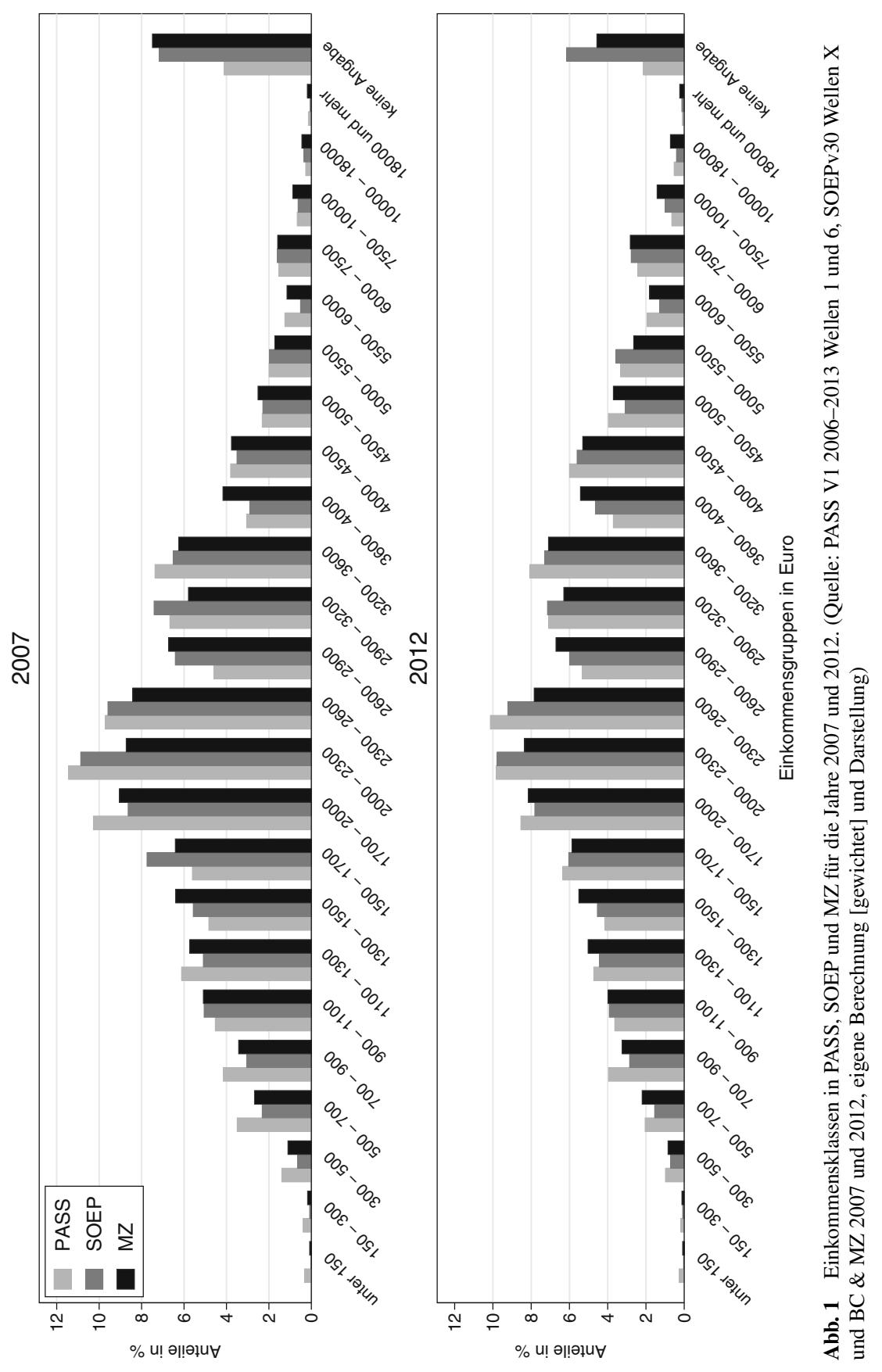


In Abb. 2 sind die Verteilungen der Äquivalenzhaushaltsnettoeinkommen der sieben Erhebungsjahre für die Stichproben des SOEP (lang gestrichelte Linie) und des PASS (durchgezogene Linie) abgebildet. Für das erste Erhebungsjahr von PASS im Jahr 2007 lässt sich erkennen, dass die Verteilung im PASS gegenüber der Verteilung im SOEP leicht nach links verschoben ist. Es ist dabei im PASS ein höherer Anteil von Haushalten mit geringem Einkommen von unter $900 €$ und ein niedriger Anteil bei den Einkommen zwischen 1000 und $1300 €$ erkennbar. Im Jahr 2008 weichen die Verteilungen vor allem für den Wertebereich von 600 bis $1300 €$ voneinander ab. Bei den Einkommen unter $600 €$ haben sich die Verteilungen stärker angepasst. Im Jahr 2009 nähern sich die Kerndichteschätzer der beiden Erhebungen weiter an. Größere Unterschiede sind hier nur noch im Wertebereich zwischen 1000 und $1200 €$ erkennbar. In den Jahren 2010 bis 2013 weisen die beiden Einkommensverteilungen die stärksten Überschneidungen auf. Kolmogorow-Smirnow-Tests auf gleiche Verteilung der Einkommen weisen in allen Wellen signifikante Differenzen auf, welche sich aber von 0,064 im Jahr 2007 auf 0,020 im Jahr 2010 zunehmend verringern. In den darauffolgenden Jahren nehmen die Differenzen wieder leicht zu (bis 0,029 im Jahr 2013).

Panelattrition tritt bei Panelstudien vor allem beim Übergang von der ersten auf die zweite Erhebungswelle auf. Davon ist das SOEP als langlaufende Panelstudie im hier untersuchten Beobachtungszeitraum nur eingeschränkt betroffen. Anders verhält es sich beim PASS, das erst im Jahr 2007 startete. Die hier beobachtete Annäherung der Kerndichteschätzer kann daher auch das Ergebnis eines Ausfallprozesses im PASS sein, der dazu führt, dass nach mehreren Erhebungswellen bestimmte Personengruppen in den zwei Stichproben nicht mehr ausreichend vertreten sind.

Um diese Überlegung zu prüfen, wird ein Vergleich der Einkommensverteilung im PASS mit der Stichprobe H des SOEP durchgeführt. Bei dieser Stichprobe handelt es sich um eine bevölkerungsrepräsentative Auffrischungsstichprobe die erstmals 2006 befragt wurde. Hier dürfte ein vergleichbarer Prozess der Panelattrition zu beobachten sein. Bei der Interpretation muss jedoch berücksichtigt werden, dass die Stichprobe H des SOEP bereits ein Jahr vor PASS zum ersten Mal befragt wurde. Die Einkommensverteilung der Stichprobe H des SOEP ist ebenfalls in Abb. 2 enthalten (kurz gestrichelte Linie). Hier deutet sich an, dass sich die Einkommensverteilungen zwischen der SOEP-Stichprobe H und der gesamten SOEP-Stichprobe stark ähnelt. Dies gilt vor allem für die Jahre 2007 und 2008. Ab dem Jahr 2009 werden die Unterschiede zur Einkommensverteilung in der PASS-Stichprobe geringer. Ab dem Jahr 2011 sind im Einkommensbereich von 500 bis $1200 €$ Abweichungen der Einkommensverteilungen zwischen der SOEP-Stichprobe $\mathrm{H}$ und den anderen beiden Stichproben zu erkennen und bleiben bis zum Jahr 2013 bestehen.

Neben der Verteilung der Einkommen kann auch die Ungleichheit der Einkommen betrachtet werden. In Abb. 3 sind die Lorenzkurven und Gini-Koeffizienten des Äquivalenzhaushaltsnettoeinkommen in SOEP und PASS für die einzelnen Jahre angegeben. Während beim SOEP der Gini-Koeffizient über die sieben Jahre relativ konstant ist, sind beim PASS stärkere Schwankungen erkennbar. Die größten Unterschiede zwischen den beiden Studien sind in den Jahren 2007 und 2008 zu erkennen. Analog zu der Entwicklung der Differenzen in den Einkommensverteilungen nehmen auch die Unterschiede in den Ungleichheitsmaßen zwischen den beiden 


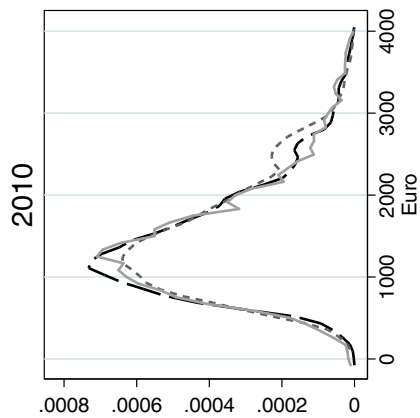

$\stackrel{\ddot{0}}{\stackrel{0}{0}}$
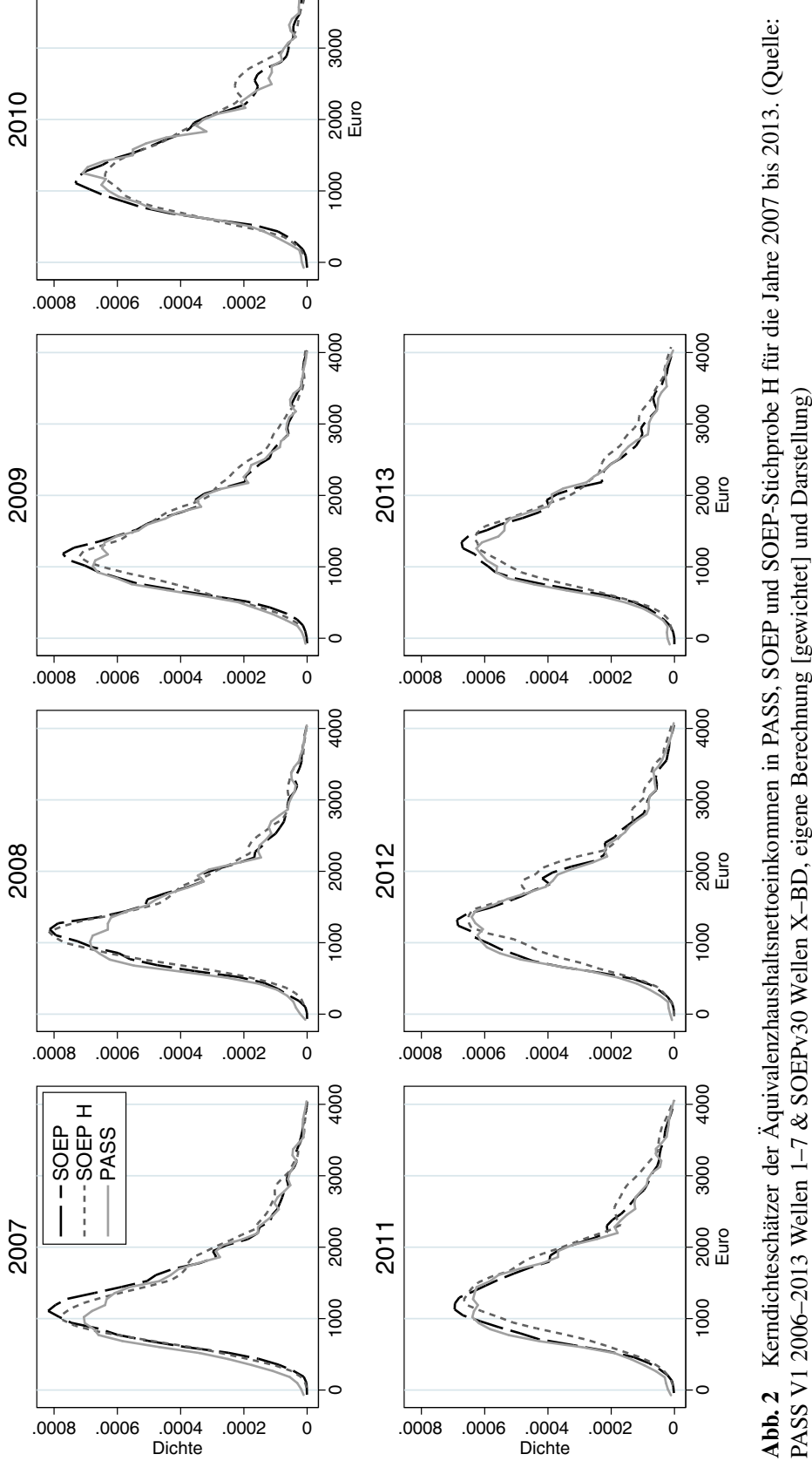

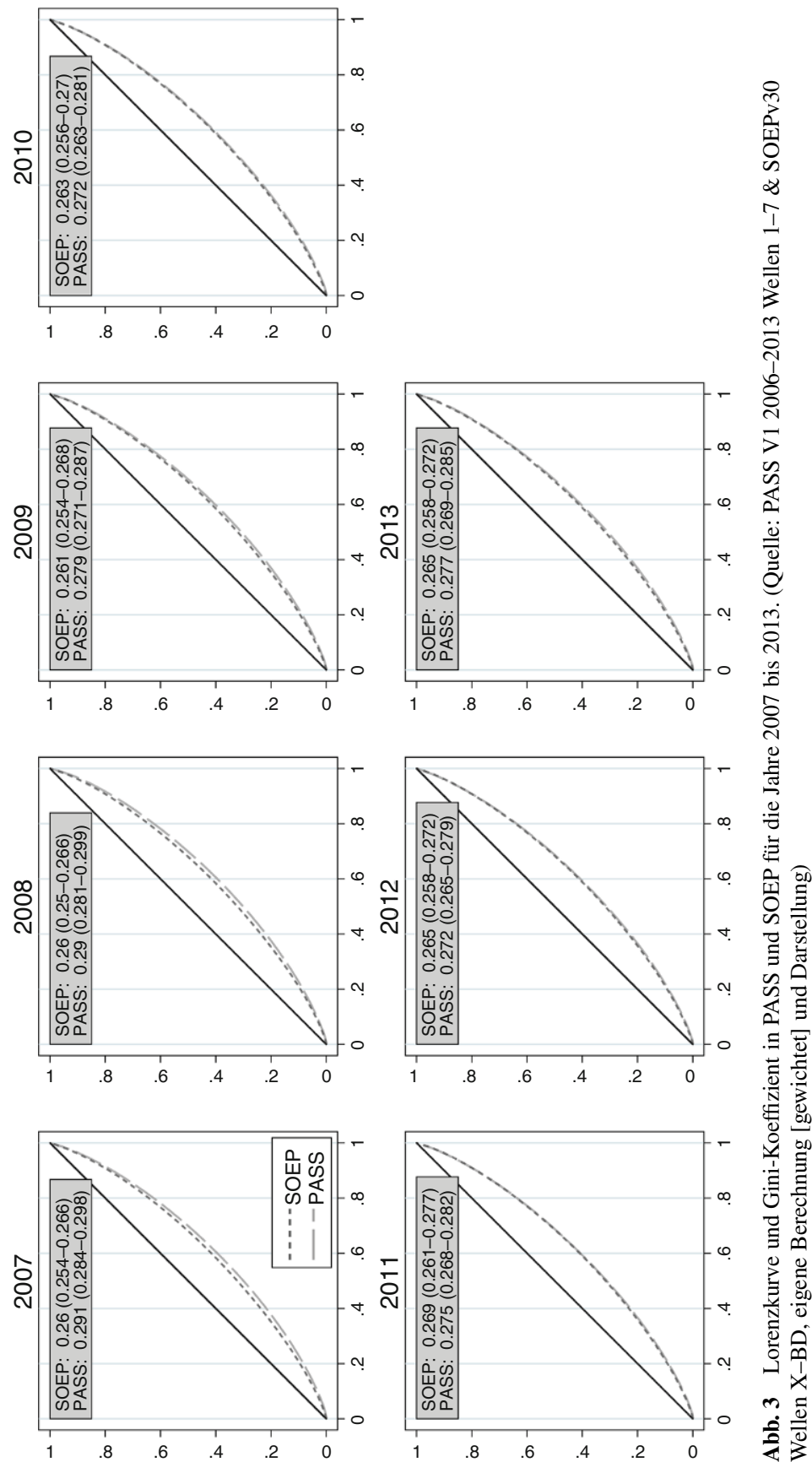
Tab. 2 Medianeinkommen, Armutsrisikoschwellenwerte und Perzentile (in Euro) in PASS, SOEP und Mikrozensus für die Jahre 2007 bis 2013

\begin{tabular}{|c|c|c|c|c|c|c|c|c|}
\hline & \multirow[t]{2}{*}{ Median } & \multirow[t]{2}{*}{$95 \%-\mathrm{KI}$} & & \multirow{2}{*}{$\begin{array}{l}\text { Armuts- } \\
\text { schwelle }\end{array}$} & \multicolumn{4}{|c|}{ Perzentile } \\
\hline & & & & & 10 & 25 & 75 & 90 \\
\hline \multicolumn{9}{|l|}{2007} \\
\hline PASS & 1212 & 1228 & 1196 & 727 & 616 & 854 & 1700 & 2333 \\
\hline SOEP & 1252 & 1260 & 1244 & 751 & 693 & 934 & 1684 & 2298 \\
\hline $\mathrm{MZ}$ & 1280 & - & - & 768 & 685 & 934 & 1740 & 2372 \\
\hline \multicolumn{9}{|l|}{2008} \\
\hline PASS & 1297 & 1314 & 1281 & 778 & 667 & 910 & 1800 & 2446 \\
\hline SOEP & 1286 & 1301 & 1271 & 772 & 706 & 965 & 1737 & 2333 \\
\hline $\mathrm{MZ}$ & 1317 & - & - & 790 & 704 & 959 & 1801 & 2441 \\
\hline \multicolumn{9}{|l|}{2009} \\
\hline PASS & 1333 & 1341 & 1325 & 800 & 700 & 952 & 1847 & 2500 \\
\hline SOEP & 1333 & 1345 & 1321 & 800 & 733 & 1000 & 1800 & 2400 \\
\hline $\mathrm{MZ}$ & 1343 & - & - & 806 & 714 & 975 & 1843 & 2500 \\
\hline \multicolumn{9}{|l|}{2010} \\
\hline PASS & 1378 & 1416 & 1340 & 827 & 730 & 1000 & 1877 & 2519 \\
\hline SOEP & 1376 & 1407 & 1344 & 825 & 750 & 1000 & 1881 & 2514 \\
\hline $\mathrm{MZ}$ & 1384 & - & - & 830 & 742 & 1006 & 1907 & 2592 \\
\hline \multicolumn{9}{|l|}{2011} \\
\hline PASS & 1392 & 1424 & 1359 & 835 & 722 & 1000 & 1894 & 2540 \\
\hline SOEP & 1400 & 1408 & 1392 & 840 & 762 & 1027 & 1933 & 2570 \\
\hline $\mathrm{MZ}$ & 1422 & - & - & 853 & 748 & 1024 & 1955 & 2662 \\
\hline \multicolumn{9}{|l|}{2012} \\
\hline PASS & 1420 & 1448 & 1391 & 852 & 748 & 1000 & 1984 & 2651 \\
\hline SOEP & 1429 & 1443 & 1415 & 857 & 777 & 1050 & 2000 & 2640 \\
\hline $\mathrm{MZ}$ & 1458 & - & - & 875 & 768 & 1047 & 2009 & 2735 \\
\hline \multicolumn{9}{|l|}{2013} \\
\hline PASS & 1467 & 1493 & 1441 & 880 & 765 & 1038 & 2000 & 2667 \\
\hline SOEP & 1467 & 1480 & 1454 & 880 & 800 & 1067 & 2000 & 2670 \\
\hline
\end{tabular}

(Quelle: PASS V1 2006-2013 Wellen 1-7, SOEPv30 Wellen X-BD, Mikrozensus 2007-2012, eigene Berechnung [gewichtet])

Datenquellen über die Jahre ab. Ab dem Jahr 2010 überlappen sich die Konfidenzintervalle, auch wenn die Differenz im Jahr 2013 wieder leicht zunimmt.

In Tab. 2 sind die Medianeinkommen als relevanter Bezugspunkt für die Armutsquotenberechnung und die sich daraus ergebenden Armutsrisikoschwellen sowie weitere Perzentile $(10 \%, 25 \%, 75 \%$ und $90 \%)$ der Verteilung dargestellt. ${ }^{22}$ Es ist zu erkennen, dass sich der Median zwischen SOEP und PASS am stärksten im Jahre 2007 (um $40 €$ ) signifikant unterscheidet. In den Jahren 2008 bis 2013 liegen die

${ }^{22}$ Für die Berechnung der Konfidenzintervalle des Medianeinkommens wird auf ein Bootstrap-Verfahren zurückgegriffen. Die Konfidenzintervalle werden nach der Perzentil-Methode ausgegeben. Dabei wird jeweils das 2,5\%-Perzentil als unterer und das 97,5\%-Perzentil als oberer Wert genommen (Shikano 2011, S. 196). Die Berechnung wird mit jeweils 1000 Replikationen durchgeführt. 
Medianeinkommen der beiden Studien sehr nah beieinander. Zum Vergleich werden für die Jahre 2007 bis 2012 die Werte auf Basis der MZ-Stichprobe angegeben. Der Median im MZ liegt in allen sechs Jahren über den Werten in SOEP und PASS. Die 10\%-Perzentile liegen im PASS in allen Jahren unter den Werten im SOEP. Die Werte im MZ liegen durchgängig dazwischen. Die 25\%-Perzentile liegen im SOEP durchgängig über denen im PASS (außer im Jahr 2010) und ähneln denen im MZ. Bei den 90\%-Perzentilen weist das PASS in den meisten Jahren hingegen höhere Werte als das SOEP auf. Hier liegen die Werte im MZ näher bei denen im PASS. Insgesamt sind die Unterschiede zwischen den drei Datenquellen als relativ gering anzusehen. Dies gilt insbesondere vor dem Hintergrund, dass kategoriale Einkommensinformationen vorliegen (in PASS bei fehlenden Antwortangaben zum exakten Einkommen und im MZ für alle Befragten), die unter entsprechenden Annahmen in metrische Informationen überführt wurden.

\subsection{Armutsmaße}

Bei der Bestimmung der Ressourcenarmut werden die Armutsquote (FGTO), die Armutslücke ( $F G T 1)$ und die Armutsintensität (FGT2) berechnet. In Abb. 4 sind diese Armutskennziffern nach der $60 \%$ Armutsrisikogrenze für die sieben untersuchten Erhebungswellen in SOEP (Dreieck) und PASS (Kreis) abgebildet. Zudem sind die 95-Prozent-Konfidenzintervalle ausgewiesen. Für die Jahre 2007 bis 2012 sind auch die Werte im MZ (Rechteck) dargestellt.

Bei der Kennziffer FGT0, welche die Armutsrisikoquote darstellt, sind deutliche Differenzen zwischen den Werten in den beiden Studien erkennbar. Diese sind besonders im Jahr 2007 ausgeprägt. Die Differenz der Punktschätzer beträgt 3,4 Prozentpunkte. Über die Jahre nähern sich die Werte einander an. Die Konfidenzintervalle überschneiden sich ab dem Jahr 2010. Im PASS werden durchgängig höhere Armutsrisikoquoten ausgegeben. Allerdings zeigen sich zwischen den beiden Datensätzen ähnliche Trends. So sind in beiden Studien ein Rückgang der Armutsrisikoquote in den Jahren 2009 sowie ein Anstieg im Zeitraum danach zu beobachten. Die im MZ ausgewiesene Armutsrisikoquote liegt durchgängig zwischen den Werten in SOEP und PASS.

Die durchgängig höheren Werte im PASS sind auch bei den weiteren Armutsmaßen FGT1 und FGT2 erkennbar. Auch hier nähern sich die Werte zwischen den Datenquellen über die Jahre einander an, allerdings vollzieht sich hier diese Entwicklung über den gesamten Beobachtungszeitraum. Überschneidungen der Konfidenzintervalle liegen ab dem Jahr 2010 (FGT1 und FGT2) vor. Die Werte des MZ liegen ebenfalls durchgängig zwischen denen in SOEP und PASS. ${ }^{23}$

\footnotetext{
23 Auch bei einer Betrachtung der Armutsmaße nach alternativen Armutsgrenzen zeigt sich ein vergleichbares Bild. Bei den Schwellenwerten von 40\%, 50\% und 70\% des Median sind deutliche Unterschiede zwischen PASS und SOEP erkennbar. Die Werte der drei FGT-Maße liegen im PASS durchgängig über denen im SOEP. Auch hier zeigt sich wieder eine Annäherung der Werte über den Beobachtungszeitraum.
} 


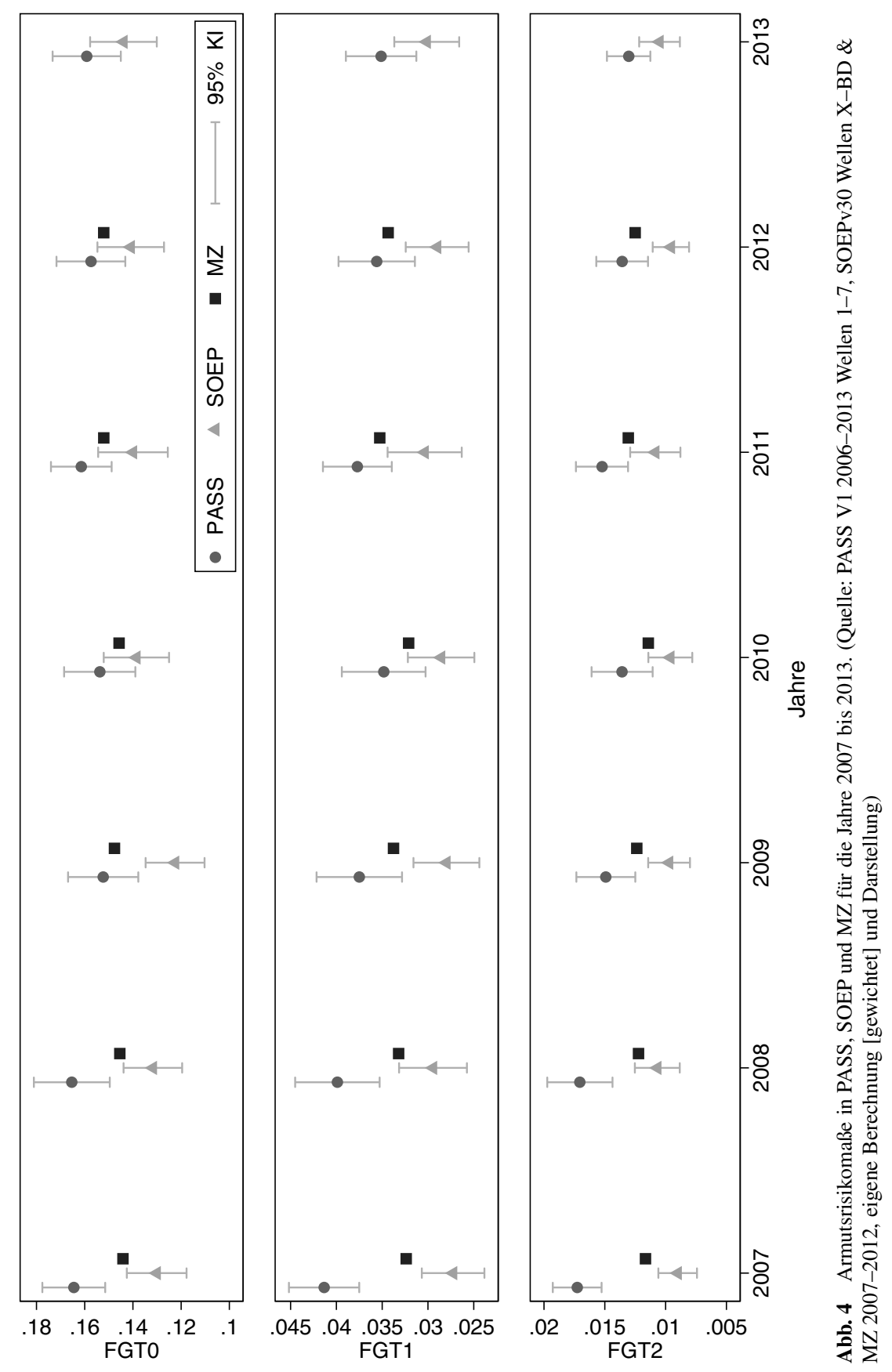




\subsection{Bevölkerungsstrukturen und Armutsrisikoquoten von Subgruppen}

Die drei Armutsmaße (FGT0 bis FGT2) unterscheiden sich zwischen den beiden Studien von 2007 bis 2009 signifikant voneinander. In einem nächsten Schritt wird versucht zu klären, wo die Quellen für diese Unterschiede liegen könnten. Um ein genaueres Bild zu erhalten, wird für das Jahr 2007 - das Erhebungsjahr mit den größten Unterschieden in den FGT-Werten - ein Blick auf die Verteilung einer Reihe zentraler soziodemographischer Charakteristika in der hochgerechneten Grundgesamtheit sowie den jeweiligen Armutsquoten in den Subpopulationen geworfen. Es werden dabei die Unterschiede zwischen SOEP und PASS nach Haushaltstyp, Haushaltsgröße, Arbeitslosengeld-II-Bezug, Wohnregion, Wohnstatus, Gesundheitszustand, Bildung, Migration, Erwerbsstatus, berufliche Stellung und Familienstand untersucht. Die Armutsrisikoquoten der Subpopulationen werden einander gegenübergestellt, um festzustellen, inwiefern die Armutsbetroffenheit der einzelnen Gruppen übereinstimmt. Um die Armutsrisikoquoten in den Subpopulationen zwischen den beiden Datensätzen besser vergleichen zu können, werden die Differenzen zur jeweils durchschnittlichen Armutsrisikoquote (PASS 16,4\%, SOEP 13,0\%) dargestellt. Dadurch soll erkennbar werden, ob es systematisch qualitativ unterschiedliche Armutsrisikogruppen gibt. Zur Kontrolle werden zusätzlich die Werte des MZ dargestellt.

Betrachtet man die Bevölkerungsstrukturen für die beiden Stichproben in Abb. 5, so zeigen sich bei den haushaltsbezogenen Merkmalen kaum signifikante Unterschiede. ${ }^{24}$ Bei den Werten, die sich durch den MZ bestimmen lassen, kommt es nur zu geringfügigen Abweichungen mit Ausnahme des Bezugs von Arbeitslosengeld-II, der im MZ etwas geringer ausfällt. Bezogen auf die Armutsrisikoquoten innerhalb der Subpopulationen ergeben sich jedoch kaum nennenswerte Unterschiede.

Betrachtet man in Abb. 6 die Bevölkerungsstruktur für die Personen ab 18 Jahren, so zeigt sich im PASS ein signifikant höherer Anteil an Personen mit „Fachhochschulabschluss“. Bei einem ,mittleren Schulabschluss mit beruflichen Bildungsabschluss“" sowie den „Hochschulabschlüssen“ hat hingegen das SOEP einen signifikant höheren Bevölkerungsanteil. Diese strukturellen Unterschiede spiegeln sich jedoch kaum in den Armutsrisikoquoten wider. Am auffälligsten - wenngleich statistisch nicht signifikant - ist hier der Unterschied in den Armutsrisikoquoten bei den Gruppen „Abitur ohne beruflicher Ausbildung“ und „Hauptschulabschluss ohne beruflicher Ausbildung“, wobei das PASS jeweils einen leicht höheren Wert aufweist.

Signifikante Unterschiede im Hinblick auf die Bevölkerungsstruktur finden sich auch beim Gesundheitszustand ${ }^{25}$, dem Migrationshintergrund und der beruflichen Stellung. Allerdings lassen sich nur bei Letzterem auch signifikante Unterschiede

\footnotetext{
${ }^{24}$ Für die Informationen zum Wohneigentum steht aus dem MZ kein Referenzwert zur Verfügung. Der Eigentümerstatus wird im MZ nur alle 5 Jahre erhoben und steht daher nicht für das Erhebungsjahr 2007 zur Verfügung.

${ }^{25}$ Eine vergleichbare Information zum Gesundheitszustand liegt im MZ für das Jahr 2007 nicht vor, da auch dieser, wie der Eigentümerstatus, nur in regelmäßigen mehrjährigen Abständen erfragt wird.
} 


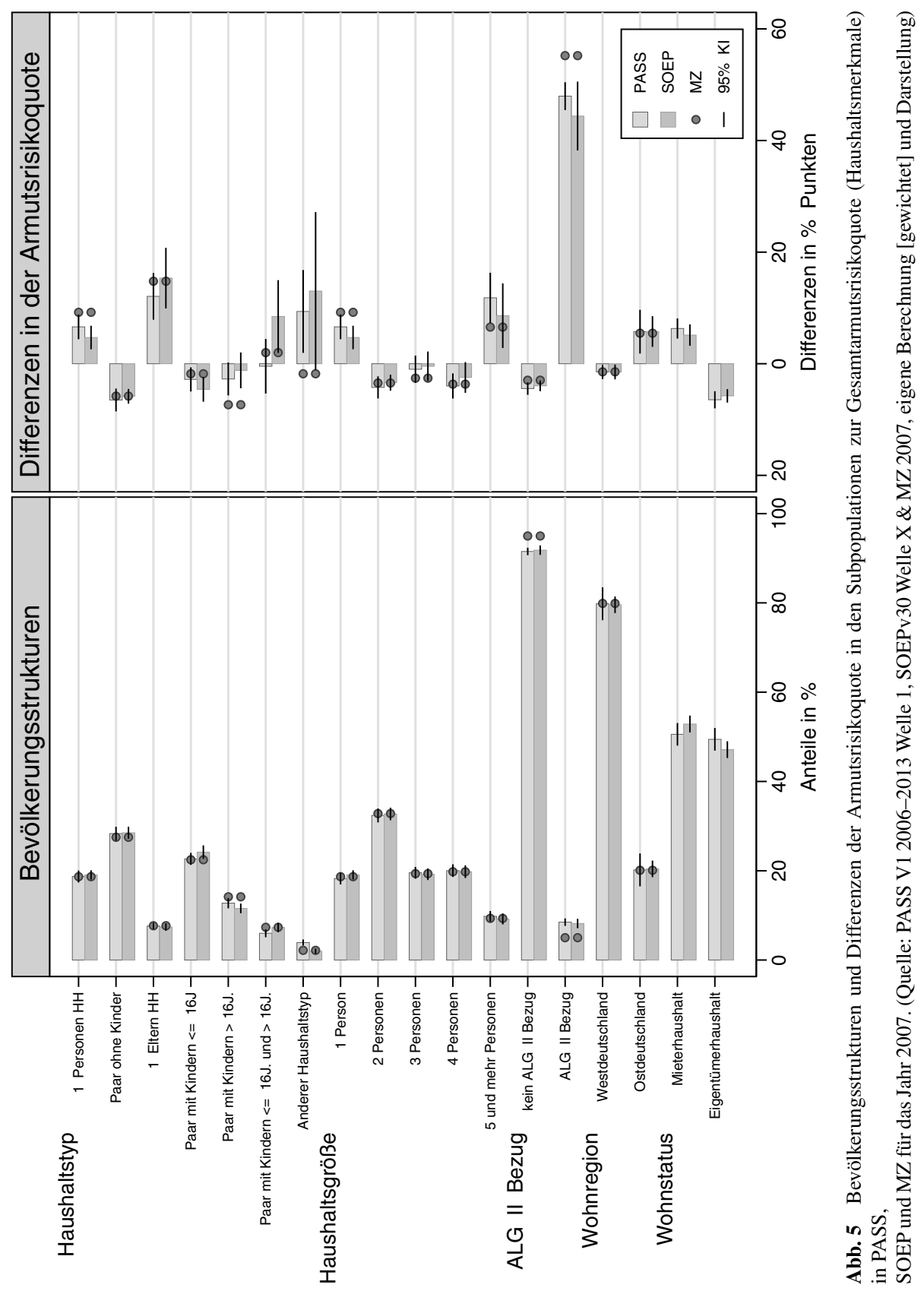




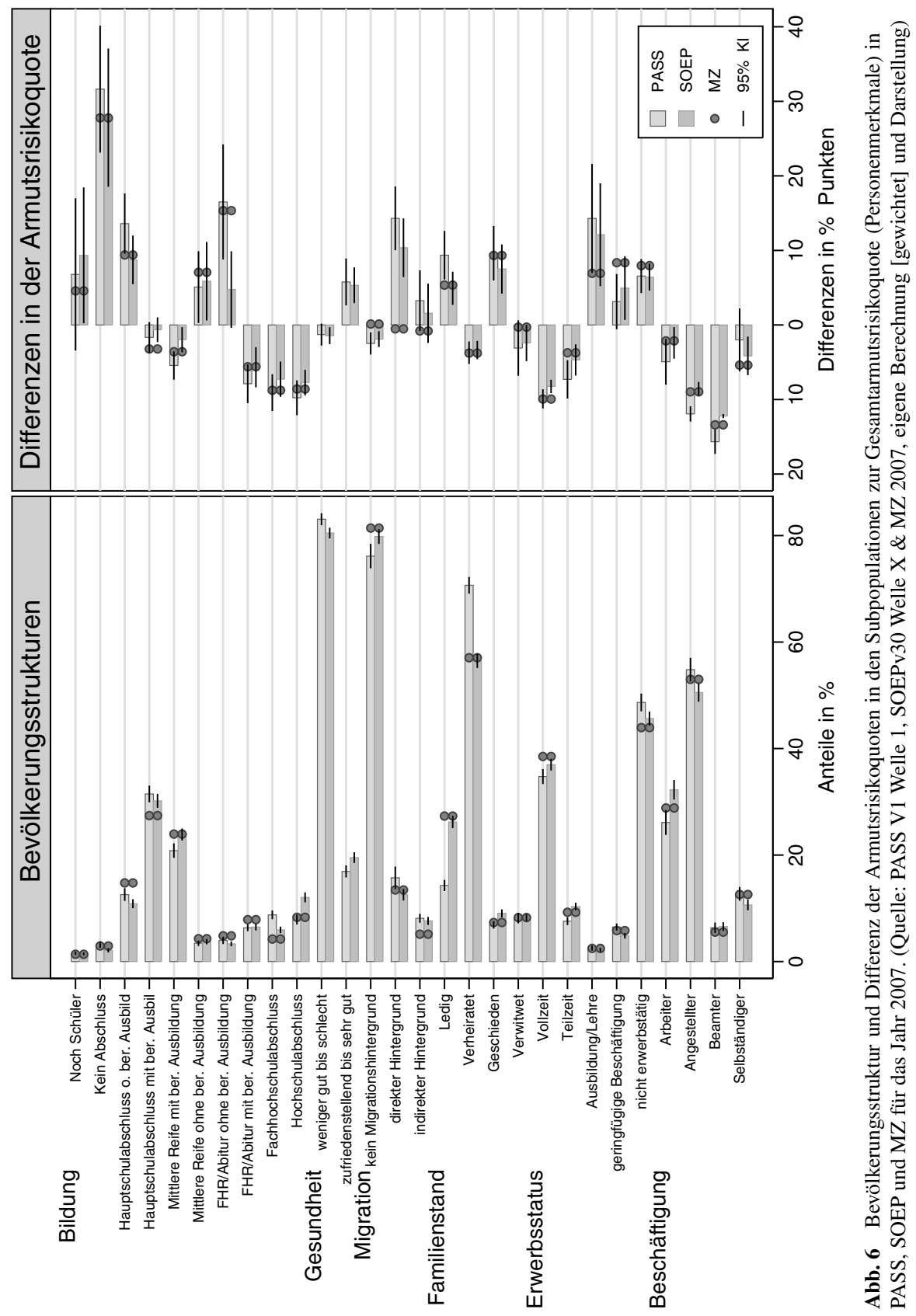


in den Abweichungen von den Armutsrisikoquoten beobachten (bei Beamten und Angestellten). Und auch beim Familienstand ergeben sich markante Unterschiede. So finden sich mehr als doppelt so viele „Ledige“ im SOEP, auch der Anteil der „Geschiedenen“ ist im SOEP signifikant höher. Im Gegenzug sind im PASS „Verheiratete" weitaus häufiger vertreten. Die Unterschiede können daraus resultieren, dass im PASS die Frage zum Familienstand in der ersten Welle eine vergleichsweise hohe Anzahl von fehlenden Werten aufweist (ca. 5\%). Die Werte im SOEP decken sich größtenteils mit denen im MZ. In den Abweichungen von den durchschnittlichen Armutsrisikoquoten lassen sich hingegen keine Unterschiede erkennen. ${ }^{26}$

\subsection{Struktur- und Armutsquoteneffekte}

Um genauere Aussagen machen zu können, ob die Unterschiede in den Armutsrisikoquoten entweder auf eine unterschiedliche Erfassung (bzw. Gewichtung) bestimmter Teilpopulationen oder auf ein unterschiedliches Armutsrisiko innerhalb der betrachteten soziodemographischen Gruppen zwischen den beiden Datensätzen zurückzuführen ist, kann die Differenz der Armutsrisikoquoten zwischen den Datensätzen in zwei Teile zerlegt werden. Bezeichnet $p$ die Gesamtarmutsrisikoquote, $\mathrm{i}$ die Bevölkerungsgruppe, $q_{i}$ den Anteil dieser Gruppe an der Bevölkerung, $\Delta q_{i}$ die Differenz der Gruppenanteile zwischen den beiden Studien und $p_{i}$ die Armutsrisikoquote der Teilpopulation, lässt sich die Differenz der Gesamtarmutsrisikoquoten zwischen SOEP und PASS beschreiben als:

$$
p^{\mathrm{SOEP}}-p^{\mathrm{PASS}}=\sum p_{i}^{\mathrm{SOEP}} \times \Delta q_{i}+\sum p_{i}^{\mathrm{PASS}} \times \Delta q_{i}
$$

Die erste Summe kann dabei als Struktureffekt interpretiert werden, die zweite als Armutsrisikoquoteneffekt. Der Struktureffekt gibt einen Hinweis darauf, ob sich die Teilpopulationen zwischen den Datensätzen unterscheiden, während der Armutsrisikoquoteneffekt einen Hinweis darauf gibt, ob in den Datensätzen unterschiedliche Anteile an Personen von Armut betroffen sind (vgl. Becker et al. 2008). Diese Zerlegung wird hier für die Merkmale vorgenommen, bei denen zwischen den beiden Datensätzen große Abweichungen in der Bevölkerungsstruktur oder der Armutsrisikoquote vorliegen. Hierzu zählen das Bildungsniveau, der Familienstand und die Form der Beschäftigung. ${ }^{27}$ In Tab. 3 sind die Ergebnisse für die Jahre 2007 und 2010

\footnotetext{
26 Vergleicht man die Bevölkerungsstrukturen über die Zeit, nähern sich die Werte auch hier einander weiter an. In 2010 - dem Jahr mit den geringsten Unterschieden in den FGT-Maßen - liegen keine signifikanten Differenzen bezüglich der Haushaltsstruktur vor. Auch in den Armutsrisikoquoten ergeben sich dann keine signifikanten Unterschiede.

Im Jahr 2010 zeigen sich keine Differenzen mehr hinsichtlich des Gesundheitszustands und des Migrationshintergrunds. Signifikante Unterschiede in der Bevölkerungsstruktur sind weiter, wenn auch nicht mehr so deutlich, bei den „Ledigen“ und den „Verheirateten“ vorhanden. Weiterhin signifikant höher ist der Anteil der Arbeiter im SOEP mit knapp 30\% gegenüber 23\% im PASS. Bei den Abweichungen von den jeweils durchschnittlichen Armutsrisikoquoten zeigen sich keine signifikanten Unterschiede mehr.

27 Die Zerlegung in Struktureffekt und Armutsrisikoquoteneffet wurde auch bei weiteren Merkmalen vorgenommen. Es zeigen sich keine weiteren nennenswerten Auffälligkeiten.
} 
Tab. 3 Differenzzerlegung in Struktur- und Armutsquoteneffekte in PASS und SOEP für die Jahre 2007 und 2010

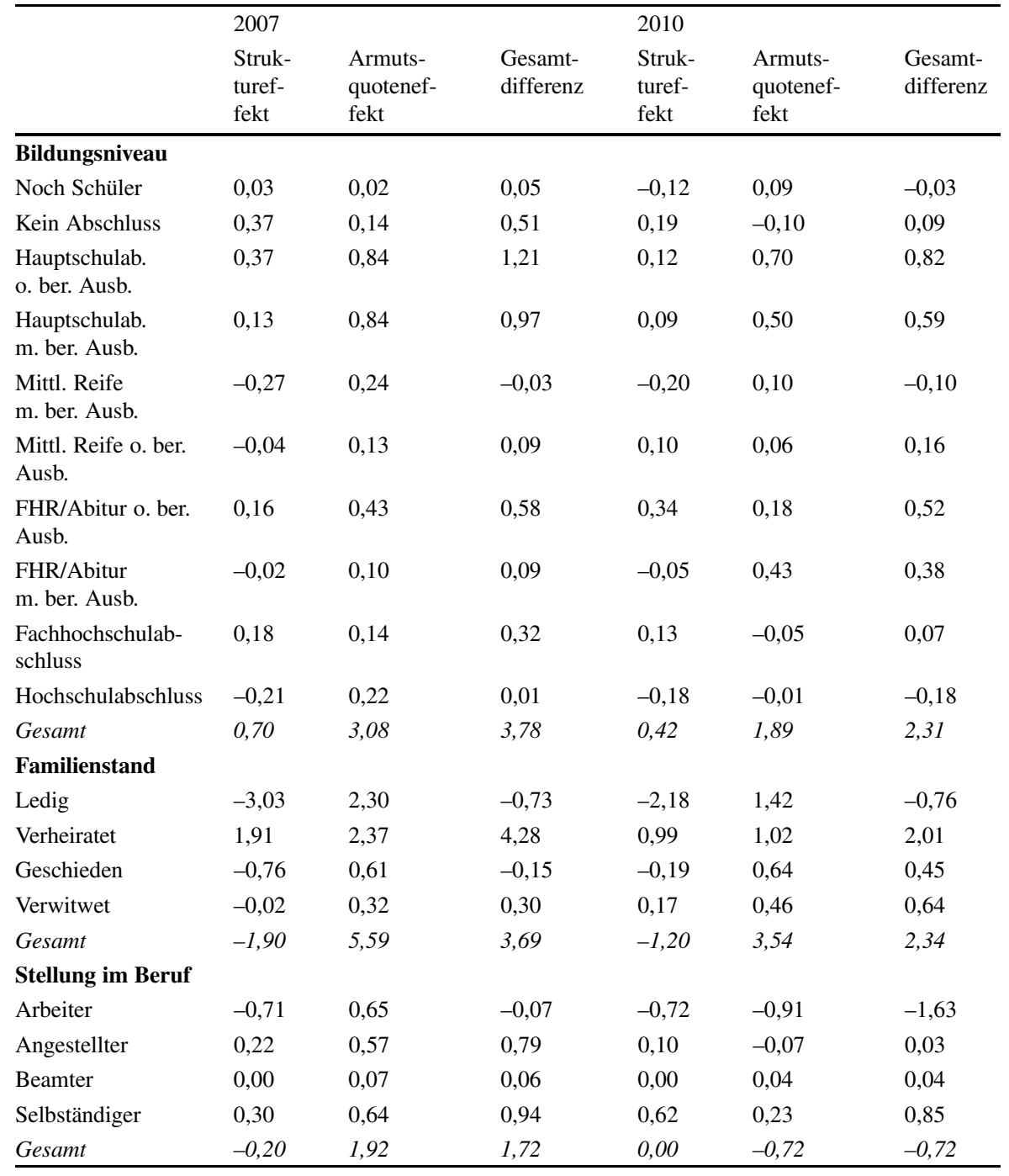

Angabe in Prozentpunkten

(Quelle: PASS V1 2006-2013 Wellen 1 und 4 \& SOEPv30 Wellen X und BA, eigene Berechnung [gewichtet])

dargestellt. Während in 2007 deutliche Unterschiede in den Armutskennziffern zu erkennen sind, kommt es bis 2010 zu einer klaren Annäherung.

Zum einen wird berechnet, wie sich die Armutsrisikoquote im PASS verändert, wenn man die Bevölkerungsanteile des SOEP unterstellt (Struktureffekt). Wäre z.B. für das Jahr 2007 die Struktur des Bildungsniveaus im PASS identisch mit der im SOEP, so würde sich die Differenz in der Armutsrisikoquote um knapp 0,7 Prozentpunkte verringern. Der Armutsrisikoquoteneffekt gibt an, um wie viel sich die 
Gesamtarmutsrisikoquote im SOEP verändert, wenn die Armutsrisikoquoten vom PASS verwendet werden. Hier ist der Effekt beim Bildungsniveau weitaus größer und beträgt 3,1 Prozentpunkte. Der Gesamteffekt ergibt sich aus der Summe von Struktureffekt und Armutsrisikoquoteneffekt. ${ }^{28}$ Der Gesamteffekt beläuft sich damit auf knapp 3,8 Prozentpunkte und ist besonders stark bei Personen mit Hauptschulabschluss (1,2 Prozentpunkte ohne berufliche Ausbildung und 1,0 Prozentpunkte mit beruflicher Ausbildung). Es ist erkennbar, dass die Differenzen zum größten Teil auf den Armutsrisikoquoteneffekt zurückzuführen sind. Die Struktureffekte sind im Vergleich dazu nur sehr gering.

Beim Familienstand wiederum ist dagegen ein deutlicher Struktureffekt erkennbar, der mit minus drei Prozentpunkten besonders stark bei der Gruppe der ,Ledigen“ ausgeprägt ist. Bei dieser Gruppe ergibt sich, wie auch bei der Gruppe der „Verheirateten“, ein starker Armutsrisikoquoteneffekt von jeweils über 2 Prozentpunkten. Der Gesamtstruktureffekt von $-1,9$ Prozentpunkten wirkt in diesem Fall dem Armutsrisikoquoteneffekt von 5,6 entgegen, so dass sich ein Gesamteffekt von 3,7 Prozentpunkten ergibt.

Betrachtet man die Zerlegung nach der beruflichen Stellung für erwerbstätige Personen ist nur ein geringer Gesamtstruktureffekt von -0,2 Prozentpunkten und ein moderater Armutsrisikoquoteneffekt von 1,9 Prozentpunkten erkennbar, wobei Beamte sich in den beiden Studien sowohl in der Struktur als auch in der Armutsrisikoquote kaum unterscheiden. Am auffallendsten sind die Ergebnisse bei Arbeitern, da der Struktur- und Armutsrisikoquoteneffekt nahezu gleich groß ist, jedoch unterschiedliche Vorzeichen aufweist. Insgesamt ergibt sich ein Gesamteffekt von 1,7 Prozentpunkten.

Vergleicht man die Gesamteffekte für die drei soziodemographischen Indikatoren über die Zeit, so nimmt die Größe dieses Wertes zwischen 2007 und 2010 jeweils ab. Macht im Jahr 2007 der Gesamteffekt beim Bildungsniveau 3,8 Prozentpunkte aus, so sinkt dieser in 2010 auf 2,3 Prozentpunkte. Dies ist vor allem auf eine Reduzierung des Armutsrisikoquoteneffekts von 3,1 auf 1,9 Prozentpunkte zurückzuführen. Der Gesamteffekt in 2010 ergibt sich weiterhin größtenteils durch die Gruppen der Personen mit Hauptschulabschluss (ohne beruflichen Abschluss 0,8 Prozentpunkt und mit beruflichen Abschluss 0,6 Prozentpunkte). Und auch beim Familienstand sinkt der Gesamteffekt von 3,7 auf 2,3 Prozentpunkte, was sich vor allem aus einer Veränderung des Armutsrisikoquoteneffekts von 5,6 auf 3,5 Prozentpunkte ergibt. Innerhalb dieser Gruppe fallen die Verheirateten auf, da die Struktur- und Armutsrisikoquoteneffekte jeweils einen Prozentpunkt ausmachten und sich ein Gesamteffekt von zwei Prozentpunkten ergibt. Bei der Form der Beschäftigung verschwindet der Struktureffekt komplett und der Gesamteffekt, der sich ausschließlich aus dem Armutsrisikoquoteneffekt ergibt, beträgt $-0,7$ Prozentpunkte. Wiederum stechen die Arbeiter hervor, deren Gesamteffekt sich auf -1,6 Prozentpunkte summiert.

\footnotetext{
28 Abweichungen der Gesamteffekte zwischen den Variablen sind auf die zugrundeliegende Population zurückzuführen. Diese können aufgrund von Einschränkungen (z. B. Personen ab 18 Jahren) oder fehlenden Werten variieren.
} 


\subsection{Armutsrisikofaktoren}

Im Folgenden wird eine multivariate Regressionsanalyse (Probit-Modell) des Armutsrisikos in Abhängigkeit von möglichen soziodemografischen Einflussfaktoren durchgeführt. Um die statistische Power zu erhöhen, wird die Analyse über gepoolte Daten der Jahre 2007 bis 2013 durchgeführt. Die Daten von SOEP und PASS werden in einem gemeinsamen Modell verwendet, wobei über Interaktionenterme separate Koeffizienten für die SOEP-Stichprobe und die PASS-Stichprobe bestimmt werden und gleichzeitig geprüft wird, ob die auftretenden Unterschiede signifikant sind. Hierdurch kann der Einfluss einzelner Determinanten für die beiden Datensätze quantifiziert und gleichzeitig miteinander verglichen werden. In dem Modell wird die Wahrscheinlichkeit geschätzt, mit der eine Person beim Vorliegen eines bestimmten Merkmals unter Berücksichtigung aller anderen Variablen als armutsrisikogefährdet gilt. Für eine einfachere Interpretation und um eine Vergleichbarkeit der Effekte mit anderen Modellen zu gewährleisten, werden Average Marginal Effekts geschätzt (vgl. Auspurg und Hinz 2011; Best und Wolf 2012). ${ }^{29}$ Jeder beobachtete Haushalt wird nur einmal in die Analyse aufgenommen, da bei Mehrpersonenhaushalten keine Unabhängigkeit der abhängigen und der unabhängigen Variablen zwischen den Haushaltsmitgliedern gegeben ist.

Zunächst zeigt sich in Tab. 4, dass der Unterschied in der Armutsrisikoquote auch im multivariaten Modell bestehen bleibt und mit -2,5 Prozentpunkten signifikant ist. Dieser Unterschied ist also auch nach Kontrolle der Verteilung der im Modell berücksichtigten unabhängigen Variablen gegeben. Die relevanten Einflussfaktoren sind in beiden Datensätzen annähernd die gleichen. Die Wirkungsrichtung stimmt bei allen signifikanten Faktoren überein. Signifikante Unterschiede in den Effektgrößen ergeben sich bei der Altersgruppe der über 64-Jährigen, Personen mit Berufsabschlüssen, der Wohnregion Ostdeutschland, Mehrgenerationen-Haushalten und nichterwerbstätigen Personen.

Die Modelle zeigen einen negativen Effekt - und damit eine geringere Wahrscheinlichkeit armutsgefährdet zu sein - für ältere Menschen, Frauen ${ }^{30}$, für Personen mit Berufsabschluss bzw. Hochschulabschluss, Besitzer von Wohneigentum und für in Partnerschaft lebende Personen. Ein höheres Risiko von Armut bedroht zu sein haben Personen mit schlechtem Gesundheitszustand, Personen mit direktem Migrationshintergrund, in den neuen Bundesländern lebende Personen, Alleinerziehende und Personen in Mehrgenerationen-Haushalten (nur im SOEP) sowie Personen die keiner Vollzeiterwerbstätigkeit nachgehen, hier insbesondere Nichterwerbstätige. Leichte Unterschiede ergeben sich jedoch, mit welcher Irrtumswahrscheinlichkeit ein Effekt signifikant von Null abweicht. Dies ist aber natürlich auch den unter-

\footnotetext{
29 Die als marginale Effekte ausgewiesenen Regressionskoeffizienten können direkt im Sinne einer prozentualen Veränderung des Armutsrisikos bei Vorliegen eines interessierenden Merkmals unter Konstanz aller anderen Einflussgrößen interpretiert werden.

30 In vielen Analysen weisen Frauen ein höheres Armutsrisiko auf als Männer. In einer bivariaten Betrachtung ist dies auch für das SOEP und das PASS zutreffend. Im multivariaten Modell unter Berücksichtigung des Erwerbsstatus zeigt sich allerdings ein für Frauen geringeres Armutsrisiko. Bei der Interpretation dieses Ergebnisses ist jedoch zu berücksichtigen, dass nur der Haushaltsvorstand in die Analyse aufgenommen wurde.
} 
Tab. 4 Korrelate des Armutsrisikos in PASS und SOEP für die Jahre 2007 bis 2013

\begin{tabular}{|c|c|c|c|c|c|c|}
\hline & \multicolumn{2}{|l|}{ PASS } & \multicolumn{2}{|l|}{ SOEP } & \multicolumn{2}{|l|}{ Differenz } \\
\hline & AME & SE & AME & SE & AME & SE \\
\hline \multicolumn{7}{|l|}{$\overline{\text { Daten }}$} \\
\hline PASS & - & - & - & - & (Ref.) & - \\
\hline SOEP & - & - & - & - & $-0,025^{* * *}$ & 0,005 \\
\hline \multicolumn{7}{|l|}{ Geschlecht } \\
\hline Männlich & (Ref.) & - & (Ref.) & - & (Ref.) & - \\
\hline Weiblich & $-0,051^{* * *}$ & 0,008 & $-0,034^{* * *}$ & 0,005 & 0,017 & 0,009 \\
\hline \multicolumn{7}{|l|}{ Alter } \\
\hline 18 bis 33 & (Ref.) & - & (Ref.) & - & (Ref.) & - \\
\hline 34 bis 49 & $-0,006$ & 0,012 & 0,010 & 0,010 & 0,016 & 0,016 \\
\hline 50 bis 64 & $-0,038^{* *}$ & 0,013 & $-0,009$ & 0,010 & 0,029 & 0,016 \\
\hline 65 und älter & $-0,154^{* * *}$ & 0,012 & $-0,118^{* * *}$ & 0,009 & $0,036^{*}$ & 0,015 \\
\hline \multicolumn{7}{|l|}{ Gesundheitszustand } \\
\hline $\begin{array}{l}\text { Zufriedenstellend bis } \\
\text { sehr gut (Ref.) }\end{array}$ & (Ref.) & - & (Ref.) & - & (Ref.) & - \\
\hline $\begin{array}{l}\text { Weniger gut bis } \\
\text { schlecht }\end{array}$ & $0,031^{* * *}$ & 0,008 & $0,026^{* * *}$ & 0,006 & $-0,006$ & 0,010 \\
\hline \multicolumn{7}{|l|}{ Migration } \\
\hline $\begin{array}{l}\text { Kein Migrationshinter- } \\
\text { grund }\end{array}$ & (Ref.) & - & (Ref.) & - & (Ref.) & - \\
\hline Direkter Hintergrund & $0,070^{* * *}$ & 0,014 & $0,083^{* * *}$ & 0,011 & 0,013 & 0,017 \\
\hline Indirekter Hintergrund & $-0,005$ & 0,012 & 0,010 & 0,009 & 0,014 & 0,015 \\
\hline \multicolumn{7}{|l|}{ Berufsbildung } \\
\hline Keinen Berufsabschluss & (Ref.) & - & (Ref.) & - & (Ref.) & - \\
\hline Berufsabschluss & $-0,094^{* * *}$ & 0,013 & $-0,059^{* * *}$ & 0,008 & $0,035^{*}$ & 0,015 \\
\hline Hochschulabschluss & $-0,146^{* * *}$ & 0,013 & $-0,117^{* * *}$ & 0,009 & 0,029 & 0,016 \\
\hline \multicolumn{7}{|l|}{ Mieterstatus } \\
\hline Mieterhaushalt & (Ref.) & - & (Ref.) & - & (Ref.) & - \\
\hline Eigentümerhaushalt & $-0,058^{* * *}$ & 0,008 & $-0,064^{* * *}$ & 0,006 & $-0,007$ & 0,010 \\
\hline \multicolumn{7}{|l|}{ Region } \\
\hline Westdeutschland & (Ref.) & - & (Ref.) & - & (Ref.) & - \\
\hline Ostdeutschland & $0,052^{* * *}$ & 0,011 & $0,082^{* * *}$ & 0,008 & $0,030^{*}$ & 0,013 \\
\hline \multicolumn{7}{|l|}{ Haushalts-Typ } \\
\hline Alleinstehende & (Ref.) & - & (Ref.) & - & (Ref.) & - \\
\hline Paar-HH & $-0,102^{* * *}$ & 0,010 & $-0,087^{* * *}$ & 0,007 & 0,015 & 0,012 \\
\hline Alleinerziehende & 0,004 & 0,016 & $0,032^{*}$ & 0,013 & 0,028 & 0,020 \\
\hline $\begin{array}{l}\text { Paar mit Kind unter } \\
16 \mathrm{~J} .\end{array}$ & $-0,081^{* * *}$ & 0,012 & $-0,081^{* * *}$ & 0,009 & $-0,001$ & 0,015 \\
\hline Paar mit Kind ab 16J. & $-0,081^{* * *}$ & 0,015 & $-0,046^{* * *}$ & 0,012 & 0,035 & 0,019 \\
\hline $\begin{array}{l}\text { Paar mit Kindern unter } \\
\text { u. ab } 16 \mathrm{~J} \text {. }\end{array}$ & $-0,006$ & 0,023 & 0,002 & 0,016 & 0,007 & 0,028 \\
\hline $\begin{array}{l}\text { Mehrgenerationen- } \\
\text { Haushalte }\end{array}$ & $-0,006$ & 0,038 & $0,113^{* *}$ & 0,037 & $0,118^{*}$ & 0,053 \\
\hline $\begin{array}{l}\text { Sonstige Kombinatio- } \\
\text { nen }\end{array}$ & $-0,035$ & 0,031 & $-0,055^{*}$ & 0,022 & $-0,020$ & 0,038 \\
\hline
\end{tabular}


Tab. 4 (Fortsetzung)

\begin{tabular}{|c|c|c|c|c|c|c|}
\hline & \multicolumn{2}{|l|}{ PASS } & \multicolumn{2}{|l|}{ SOEP } & \multicolumn{2}{|l|}{ Differenz } \\
\hline & AME & SE & AME & SE & AME & SE \\
\hline \multicolumn{7}{|c|}{ Erwerbs- bzw. Nichtstatus } \\
\hline Vollzeit & (Ref.) & - & (Ref.) & - & (Ref.) & - \\
\hline Teilzeit & $0,045^{* * *}$ & 0,008 & $0,064^{* * *}$ & 0,007 & 0,019 & 0,011 \\
\hline Ausbildung/Lehre & $0,139^{* * *}$ & 0,023 & $0,110^{* * *}$ & 0,015 & $-0,029$ & 0,028 \\
\hline $\begin{array}{l}\text { Geringfügige Beschäfti- } \\
\text { gung }\end{array}$ & $0,173^{* * *}$ & 0,013 & $0,177^{* * *}$ & 0,012 & 0,003 & 0,018 \\
\hline Nicht erwerbstätig & $0,253^{* * *}$ & 0,009 & $0,210^{* * * *}$ & 0,007 & $-0,042^{* * *}$ & 0,012 \\
\hline \multicolumn{7}{|l|}{ Erhebungsjahr } \\
\hline 2007 & (Ref.) & - & (Ref.) & - & (Ref.) & - \\
\hline 2008 & 0,014 & 0,007 & $0,011^{*}$ & 0,005 & $-0,004$ & 0,009 \\
\hline 2009 & $0,016^{*}$ & 0,008 & 0,005 & 0,005 & $-0,011$ & 0,009 \\
\hline 2010 & $0,022^{*}$ & 0,010 & $0,019^{* * *}$ & 0,005 & $-0,004$ & 0,011 \\
\hline 2011 & $0,017^{*}$ & 0,008 & $0,029^{* * *}$ & 0,005 & 0,012 & 0,010 \\
\hline 2012 & 0,007 & 0,008 & $0,020^{* * *}$ & 0,005 & 0,013 & 0,010 \\
\hline 2013 & $0,026^{* *}$ & 0,009 & $0,018^{* *}$ & 0,005 & $-0,009$ & 0,010 \\
\hline Fallzahl & 65.076 & - & 78.847 & - & 143.923 & - \\
\hline Pseudo-R ${ }^{2}$ & 0,228 & - & 0,232 & - & 0,232 & - \\
\hline
\end{tabular}

Effekte als durchschnittliche marginale Effekten (AME) mit Standardfehlern (SE)

Signifikanzniveaus: * $p<0,05 ;{ }^{* *} p<0,01 ;{ }^{* * *} p<0,001$

(Quelle: PASS V1 2006-2013 Wellen 1-7 \& SOEPv30 Wellen X-BD, eigene Berechnung [gewichtet])

schiedlichen hohen Fallzahlen geschuldet. Insgesamt zeigt sich, dass man bei dieser multivariaten Analyse mit beiden Datensätzen zu sehr vergleichbaren Ergebnissen kommt.

\subsection{Permanente Armut und Übergangsraten}

Um die spezifischen Vorteile zu nutzen, die sich durch die Paneldatenstruktur der beiden Studien ergeben, wird abschließend noch ein Blick auf das Ausmaß dauerhafter Armut und die Dynamik innerhalb der Armutspopulationen geworfen. Aus einer zeitlichen Perspektive lässt sich zwischen Personen unterscheiden, die über einen längeren Zeitraum nie, vorübergehend oder dauerhaft von Armut betroffen sind. Während bei vorübergehender Armut - auch transitorische Armut genannt eine zumindest zeitweise Teilhabe am gesellschaftlichen Zusammenleben möglich ist, stellt permanente Armut eine extreme Ausgrenzung der betroffenen Personen dar.

In Tab. 5 sind die beobachteten Jahre in Armut im Zeitraum von 2007 bis 2013 wiedergegeben. Grundlage ist ein balanciertes Panel, d.h. es gehen nur Personen in die Analyse ein, für die in allen sieben Jahren ein realisiertes Interview vorliegt. Der Anteil an Personen, die in den sieben Jahren nie von Armut betroffen sind, liegt im SOEP deutlich über dem im PASS. Die Konfidenzintervalle der beiden Werte überlappen sich nur knapp. Im PASS liegen die Anteile der Personen, die 1 bis 2 Jahre, 3 bis 4 Jahre oder 5 bis 7 Jahre armutsgefährdet sind, höher als im SOEP. 
Tab. 5 Anzahl an Jahren in Armut in den sieben beobachteten Jahren (2007-2013) in PASS und SOEP

\begin{tabular}{lllllll}
\hline In Armut & PASS & & \multicolumn{3}{l}{ SOEP } \\
& Anteil & $95 \%$-KI & & Anteil & $95 \%$-KI & \\
\hline Kein Jahr & 64,7 & 61,4 & 68,1 & 71,1 & 67,4 & 74,8 \\
1 bis 2 Jahre & 18,4 & 15,8 & 21,0 & 15,8 & 12,5 & 19,1 \\
3 bis 4 Jahre & 7,2 & 5,4 & 9,1 & 5,5 & 4,2 & 6,8 \\
5 bis 7 Jahre & 9,7 & 7,4 & 11,9 & 7,7 & 5,7 & 9,6 \\
\hline
\end{tabular}

Anteile in Prozent

(Quelle: PASS V1 2006-2013 Wellen 1-7 \& SOEPv30 Wellen X-BD, eigene Berechnung [gewichtet])

Die Gruppen weichen aber nicht stark voneinander ab, d.h. in beiden Erhebungen wird ein vergleichbar hohes Ausmaß an transitorischer als auch permanenter Armut gemessen. Diese Ergebnisse sagen aber noch nicht viel über die Dynamik im Armutsbereich aus. Denn Personen mit mehreren Jahren Armutserfahrungen können entweder längere Armutsepisoden am Stück oder häufige Wechsel in und aus dem Armutsbereich aufweisen.

Daher wird zusätzlich ein Blick auf die Dynamik im Armutsbereich geworfen. Die Dynamik gibt Auskunft darüber, wie innerhalb einer Gesellschaft die Chancen und Risiken der Einkommensverbesserung bzw. -verschlechterung sind. Die Veränderung von Einkommenspositionen im Zeitverlauf gibt einen Hinweis darauf, ob defizitäre Positionen überwunden werden können und welchem Risiko Haushalte und Personen ausgesetzt sind, in unzureichende Einkommenslagen abzusteigen. Wir konzentrieren uns hier auf eine Analyse der Aufstiege aus bzw. Abstiege in den Armutsrisikobereich. Das PASS, das durch seine spezifische Stichprobenzusammensetzung eine hohe Fallzahl von Haushalten und Personen mit niedrigen Einkommen aufweist, ist unter anderem für solche Analysen konzipiert worden.

Es wird hierfür untersucht, wie hoch der Anteil an Personen ist, die von einem Jahr zum nächsten den Armutsbereich verlassen bzw. in den Armutsbereich gewechselt sind. Tab. 6 zeigt, dass sowohl die Aufstiegsraten als auch die Abstiegsraten in den meisten Jahren im PASS höher liegen. Signifikante Unterschiede liegen aber nur bei den Personen mit einem Abstieg in Armutsrisiko für die Jahre 2008 und 2012 vor.

\section{Fazit}

In der Armutsberichterstattung werden zur Beschreibung der aktuellen Lage häufig deskriptive Statistiken herangezogen. Um Aussagen über das Ausmaß und die Veränderung von Armut machen zu können, bedarf es einer validen Datengrundlage. Hierfür eignen sich Informationen zum Einkommen eines Haushaltes, die im Rahmen von Haushaltpanelbefragungen erhoben werden. Allerdings sind solche Informationen immer mit bestimmten Unsicherheiten behaftet und potenziellen Fehlerquellen ausgesetzt. So kann es sein, dass sich die Ergebnisse auf Basis zweier Studien, die mit vergleichbaren Messinstrumenten für eine vergleichbare Grundgesamtheit für einen vergleichbaren Zeitraum Informationen erheben, voneinander abweichen. Dies kann auch für Maße zur Beschreibung von Armut gelten. Um die Aussagekraft 
Tab. 6 Aufstiege aus dem Armutsbereich und Abstiege in den Armutsbereich in PASS und SOEP für die Jahre 2008 bis 2013

\begin{tabular}{lllllll}
\hline & $\begin{array}{l}\text { PASS } \\
\text { Anteil }\end{array}$ & $95 \%$-KI & & $\begin{array}{l}\text { SOEP } \\
\text { Anteil }\end{array}$ & $95 \%$-KI & \\
\hline Aufstiege & & & & & \\
2008 & 40,2 & 36,2 & 44,1 & 31,6 & 26,1 & 37,0 \\
2009 & 38,4 & 33,3 & 43,4 & 39,5 & 33,5 & 45,5 \\
2010 & 36,6 & 31,0 & 42,2 & 26,8 & 21,4 & 32,1 \\
2011 & 39,7 & 34,0 & 45,4 & 31,5 & 25,9 & 37,0 \\
2012 & 36,2 & 32,0 & 40,4 & 30,4 & 25,1 & 35,7 \\
2013 & 35,4 & 30,6 & 40,1 & 31,8 & 26,3 & 37,2 \\
Abstiege & & & & & 4,5 & 6,5 \\
2008 & 9,3 & 7,9 & 10,6 & 5,5 & 3,5 & 6,0 \\
2009 & 6,6 & 5,5 & 7,6 & 4,7 & 4,8 & 7,1 \\
2010 & 7,5 & 6,3 & 8,8 & 6,0 & 4,7 & 7,3 \\
2011 & 5,3 & 4,1 & 6,6 & 6,0 & 3,0 & 4,6 \\
2012 & 6,1 & 5,0 & 7,3 & 3,8 & 4,3 & 6,0 \\
2013 & 6,6 & 5,5 & 7,8 & 5,1 & &
\end{tabular}

Anteile in Prozent

(Quelle: PASS V1 2006-2013 Wellen 1-7 \& SOEPv30 Wellen X-BD, eigene Berechnung [gewichtet])

von Ergebnissen zu Armut auf Basis dieser Datengrundlagen besser einschätzen zu können, bietet sich ein Vergleich der Ergebnisse auf Basis unterschiedlicher Studien an.

In dem hier untersuchten Fall können Unterschiede in den Einkommensverteilungen und teilweise signifikante Abweichungen zwischen PASS und SOEP in den Armutsmaßen FGT0, FGT1 und FTG2 vor allem im Zeitraum 2007 bis 2009 den ersten drei Erhebungswellen des PASS - festgestellt werden. Als Gründe hierfür können verschiedene Quellen vermutet werden, die sich nicht alle abschließend überprüfen lassen. So können beim Stichprobenverfahren sowie beim Messverfahren studienspezifischen Unterschiede auftreten. Erklärungen lassen sich teilweise durch spezifische Eigenschaften der Studien finden. So sind auf Grund des Stichprobendesigns im PASS mehr Fälle im unteren Einkommensbereich vorhanden. Im SOEP hingegen stehen insgesamt mehr Fälle zur Verfügung. Das Studiendesign sowie bestimmte Ausfallprozesse werden in der Gewichtung berücksichtigt, wodurch Aussagen über die Gesamtpopulation möglich sein sollen.

Über die Zeit kann eine Annäherung des erfassten Armutsrisikos in den beiden Studien beobachtet werden. Hierfür können zwei Erklärungen dienen. Entweder kann es aufgrund der schlechteren Datenqualität bei den Einkommensangaben in den ersten Erhebungswellen zu einer Verzerrung der Armutsrisikoquote kommen. Dies würde bedeuten, dass die Armutsrisikoquote im PASS im Jahr 2007 weiter vom wahren Wert entfernt liegt und sich mit zunehmenden Erhebungswellen dem wahren Wert nähert. Dies könnte u.a. daran liegen, dass Befragte in einer ersten Welle mit dem Ablauf der Befragung, den befragten Inhalten als auch mit dem Interviewer nicht vertraut sind. Dies führt häufig zu mehr Antwortverweigerungen, mehr gerundeten Angaben oder auch falschen Antworten aufgrund von Verständ- 
nisproblemen. In den folgenden Wellen nehmen diese Probleme ab und die Qualität der Antwortangaben steigt, da u.a. Befragte Unterlagen wie Lohnbescheide für die Interviews heranziehen und abgefragte Beträge direkt aus Lohnzetteln übertragen. Hierfür spricht, dass in den Jahren 2007 und 2008 die Armutsmaße im SOEP näher an denen im ebenso bevölkerungsrepräsentativen MZ liegen.

Andererseits kann es auch zu einer Verzerrung der Armutsrisikoquote in Folge der Panelausfälle kommen. Bestimmte Gruppen, die von Armut stärker betroffen sind, nehmen in den Folgebefragungen weniger wahrscheinlich teil. Falls diese Ausfälle mit bestimmten Ereignissen wie Jobverlust oder Veränderungen der Haushaltskonstellation - Ereignisse, die wiederum das Armutsrisiko erhöhen können, - verbunden sind, wird dies durch die Gewichtung eventuell nicht hinreichend korrigiert und die Armutsrisikoquote in der Folge unterschätzt. Dann würde sich die Armutsrisikoquote im PASS aufgrund der Ausfälle in den Wellen nach der Ersterhebung zunehmend vom wahren Wert entfernen. ${ }^{31}$ Für diese Annahme spricht, dass in PASS im Jahr 2011 eine Auffrischung der Stichprobe stattgefunden hat und in diesem Jahr auch ein Wiederanstieg der Armutsrisikoquote zu erkennen ist.

Welche der Erklärungen zutrifft, lässt sich hier nicht abschließend klären. Es lassen sich darüber hinaus jedoch auch viele Ergebnisse finden, die sich zwischen den beiden Studien stark ähneln. So ist die Struktur des Armutsrisikos in großen Teilen vergleichbar, wenn auch die Armutsrisikoquoten im PASS durchschnittlich höher liegen. Bei einem Vergleich der Ergebnisse multivariater Analysen lassen sich große Gemeinsamkeiten zwischen den beiden Panelstudien feststellen. Bei der Dauer und Dynamik von Armut liegen nur wenig signifikante Unterschiede vor, im PASS wird aber eine durchweg höhere Mobilität in Armut und aus Armut heraus beobachtet.

Es muss aber auch festgehalten werden, dass bei einigen Analysen eine recht hohe statistische Unsicherheit vorliegt. Dadurch können auch größere Unterschiede zwischen den Studien nicht als statistisch gesichert eingestuft werden. Dies kann gerade bei Längsschnittanalysen aufgrund sinkender Fallzahlen ein Problem darstellen.

Betrachtet man die Gemeinsamkeiten und Unterschiede in den Ergebnissen, so lässt sich insgesamt ein sehr ähnliches Bild der Armut in Deutschland in den Jahren 2007 bis 2013 gewinnen. Die sozialpolitischen Implikationen zum Thema Armut, die auf Basis der beiden Studien abgeleitet werden, dürften sicherlich sehr vergleichbar ausfallen und die Gefahr, dass konträre Empfehlungen ausgesprochen würden, ist als gering einzustufen.

Für ArmutsforscherInnen, die auf die beiden Datenquellen SOEP und PASS zurückgreifen möchten, kann folgende Empfehlung gegeben werden: Beide Datenquellen sind grundsätzlich geeignet das Ausmaß an Armut(-srisiko) und Armutsmobilität in Deutschland angemessen zu beschreiben. Zu berücksichtigen ist jedoch, dass bei Längsschnittstudien besonders in den ersten Jahren nach Studienbeginn Prozesse stattfinden, die auch die Ergebnisse von Armutsanalysen beeinflussen können. Inwieweit dies allerdings zu verzerrten Ergebnissen führt, ist hier nicht eindeutig zu klären. Zudem können geringe Fallzahlen die Interpretation von zeitlichen Entwick-

31 Die relativ hohe Attrition im PASS ist dabei auch auf die spezifische Stichprobenzusammensetzung zurückzuführen. 
lungen erschweren, da sich diese häufig im Bereich der statistischen Unsicherheit bewegen.

In dieser Untersuchung konnte nicht im Detail auf die einzelnen potenziellen Fehlerquellen im gesamten Prozess der Erhebung und Aufbereitung der Daten eingegangen werden. Es ist sicher sinnvoll, die im Rahmen der Total-Survey-ErrorForschung benannten potenziellen Fehlerquellen auf ihren Einfluss auf die Ergebnisse von Armutsanalysen genauer hin zu untersuchen.

Insgesamt kann aus den Ergebnissen dieser Untersuchung eine hohe Bedeutung für die Verwendung verschiedener Datenquellen für die Analyse von Armut abgeleitet werden. Die Replikation von Forschungsarbeiten ermöglicht zudem, Ergebnisse besser einschätzen zu können und sich die Grenzen der Interpretation bewusst zu machen. Basierend auf den Erkenntnissen dieser Untersuchung empfiehlt es sich, Vergleiche zwischen verschiedenen Studien, die sich zur Analyse von Armut eignen, zu wiederholen, um so ein noch differenziertes Bild des Einflusses studienspezifischer Gegebenheiten zu erhalten. Zudem sollten wiederholt vergleichbare Arbeiten wie diese zur Erfassung der Datenqualität durchgeführt werden, um möglichst frühzeitig potentielle Defizite in den Mikrodatenbasen identifizieren zu können und diese zu korrigieren.

\section{Anhang}

\section{Fragetext zum Screener-Einkommen in SOEP und PASS}

Der Originaltext der Frage zum Screener-Einkommen im SOEP lautet:

- Wenn man mal alle Einkünfte zusammennimmt:

- Wie hoch ist das monatliche Haushaltseinkommen aller Haushaltsmitglieder heute?

- Bitte geben Sie den monatlichen Netto-Betrag an, also nach Abzug von Steuern und Sozialabgaben.

- Regelmäßige Zahlungen wie Renten, Wohngeld, Kindergeld, BAföG, Unterhaltszahlungen usw. rechnen Sie bitte dazu!

- Falls nicht genau bekannt:

- Bitte schätzen Sie den monatlichen Betrag. ... Euro im Monat

Der Originaltext der Frage zum Screener-Einkommen ${ }^{32}$ im PASS lautet:

\footnotetext{
32 Personen, die diese Frage mit „,weiß nicht“ oder „keine Angabe“ beantwortet haben, bekommen im Anschluss eine Nachfrage, bei der Sie ihr Einkommen in Kategorien angeben können. Dabei werden die Kategorien in drei Schritten immer weiter verengt. Die Kategorien im ersten Schritt lauten: ,weniger als $1000 €$ “ und „,1000€ und mehr“. Im zweiten Schritt: ,unter $500 €$ “, ,500 bis $750 €$ “ und „,mehr als $750 €$ “ bzw. ,weniger als $3000 €$ “ und ,,3000€ und mehr“. Im dritten Schritt: ,,unter $1500 €$ “, „,1500 bis $2000 €$ “ und „2000€ und mehr“ bzw. ,unter $4000 €$ ", „,4000 bis $5000 €$ “ und ,5000€ und mehr“. Beim SOEP werden in vergleichbarer Weise bei einer fehlenden Antwortangabe 10 verschiedene Schwellenwerte schrittweise den Befragten angeboten.
} 
- Wenn Sie einmal alle Einkünfte Ihres Haushalts zusammenrechnen, auch die bereits genannten: Wie hoch war das Netto-Einkommen Ihres Haushalts insgesamt im letzten Monat?

- Ich meine damit die Summe der Erwerbseinkommen aller Haushaltsmitglieder, aller Sozialleistungen, wie z. B. Kindergeld, Arbeitslosengeld [inklusive der Kosten der Unterkunft], Renten oder Pensionen und aller sonstigen Einkünfte, die nach Abzug von Steuern und Sozialversicherungsbeiträgen übrig bleibt.

- Interviewer: Bei Einkommen aus selbständiger Tätigkeit bitte den für den persönlichen Verbrauch entnommenen Betrag verwenden.

- Euro ... pro Monat

Open Access Dieser Artikel wird unter der Creative Commons Namensnennung 4.0 International Lizenz (http://creativecommons.org/licenses/by/4.0/deed.de) veröffentlicht, welche die Nutzung, Vervielfältigung, Bearbeitung, Verbreitung und Wiedergabe in jeglichem Medium und Format erlaubt, sofern Sie den/die ursprünglichen Autor(en) und die Quelle ordnungsgemäß nennen, einen Link zur Creative Commons Lizenz beifügen und angeben, ob Änderungen vorgenommen wurden.

\section{Literatur}

Atkinson AB, Marlier E (2010) Income and living conditions in Europe. Eurostat Statistical Book. Luxembourg: Publications Office ot the European Union.

Auspurg K, Hinz T (2011) Gruppenvergleiche bei Regressionen mit binären abhängigen Variablen: Probleme und Fehleinschätzungen am Beispiel von Bildungschancen im Kohortenverlauf. Z Soziol 40(1):62-73

Becker I, Grabka MM, Hauser R, Westerheide P (2008) Integrierte Analyse der Einkommens- und Vermögensverteilung. Abschlussbericht zur Studie im Auftrag des Bundesministeriums für Arbeit und Soziales. Bundesministerium für Arbeit und Soziales, Bonn

Berg M, Cramer R, Dickmann C, Gilberg R, Jesske B, Kleudgen M, Bethmann A, Fuchs B, Hubert M, Trappmann M (2014) Codebuch und Dokumentation des ,Panel Arbeitsmarkt und soziale Sicherung“ (PASS) Datenreport Welle 7. FDZ-Datenreport 02/2014, Nürnberg. http://doku.iab.de/fdz/reporte/ 2014/DR_02-14_I.pdf. Zugegriffen: 18. Apr. 2016

Best H, Wolf C (2012) Modellvergleich und Ergebnisinterpretation in Logit- und Probit-Regressionen. Kolner Z Soz Sozpsychol 64:377-395

Bethmann A, Fuchs B, Wurdack A (2013) User Guide 'Panel Arbeitsmarkt und soziale Sicherung' (PASS) Wave 6. FDZ-Datenreport 06/2013, Nürnberg. http://doku.iab.de/fdz/reporte/2013/DR_07-13.pdf. Zugegriffen: 15. Mai 2016

Blumenberg MS, Roßmann J, Gummer T (2013) Bericht zur Datenqualität der GLES 2009. GESIS-Technical Reports 2013|14

Boehle M (2015) Armutsmessung mit dem Mikrozensus. Methodische Aspekte und Umsetzungen für Querschnitts- und Trendanalysen. Gesis Papers 2015/16, Mannheim. http://www.gesis.org/fileadmin/ upload/forschung/publikationen/gesis_reihen/gesis_papers/GESIS-Papers_2015-16.pdf. Zugegriffen: 20. Okt. 2016

Bundesministerium für Arbeit und Soziales (Hrsg) (2017) Lebenslagen in Deutschland. Der Fünfte Armuts- und Reichtumsbericht der Bundesregierung. http://www.armuts-und-reichtumsbericht.de/DE/ Bericht/Der-fuenfte-Bericht/fuenfter-bericht.html. Zugegriffen: 20. Jan. 2018

Van Buuren S, Groothuis-Oudshoorn K (2000) Multivariate Imputation by Chained Equations: MICE V1.0 User's manual. TNO Report PG/VGZ/00.038. TNO Prevention and Health, Leiden. http:// www.stefvanbuuren.nl/publications/MICE\%20V1.0\%20Manual\%20TNO00038\%202000.pdf. Zugegriffen: 15. Mai 2016

Foster J, Greer J, Thorbecke E (1984) A class of decomposable poverty measures. Econometrica 52(3):761-766

Frick JR, Grabka MM (2005) Item-non-response on income questions in panel surveys: incidence, imputation and the impact on the income distribution. Wirtsch Sozialstatist Arch 89(1):49-61

Frick JR, Grabka MM (2010) Item non-response and imputation of annual labor income in panel surveys from a cross-national perspective. In: Harkness JA, Edwards B, Braun M, Johnson TP, Lyberg LE, 
Mohler PP, Pennell B-E, Smith T (Hrsg) Survey methods in multicultural, multinational, and Multiregional contexts. Wiley, New York, S 355-374

Frick JR, Goebel J, Grabka MM, Groh-Samberg O, Wagner GG (2007) Zur Erfassung von Einkommen und Vermögen in Haushaltssurveys: Hocheinkommensstichprobe und Vermögensbilanz im SOEP. DIW Data Documentation 19, Berlin. http://www.diw.de/documents/publikationen/73/diw_01.c.57345.de/ diw_sp0019.pdf. Zugegriffen: 11. Okt. 2016

Frick JR, Grabka MM, Smeeding T, Tsakloklu P (2010) Distributional effects of imputed rents in five European countries. J Hous Econ 19:167-179

Frick JR, Grabka MM, Groh-Samberg O (2012) Dealing with incomplete household panel data in inequality research. Sociol Methods Res 41(1):89-123

Gerhardt A, Habenicht K, Munz E (2009) Analysen zur Einkommensarmut mit Datenquellen der amtlichen Statistik. In: Information und Technik Nordrhein-Westfalen (Hrsg) Statistische Analysen und Studien NRW, Bd. 58. Information und Technik Nordrhein-Westfalen, Düsseldorf (http://www.it.nrw. de/statistik/analysen/stat_studien/2009/band_58/z089200954.pdf. Zugegriffen: 16. April 2016)

Goebel J, Habich R, Krause P (2009) Zur Angleichung von Einkommen und Lebensqualität in Deutschland. Vierteljahresh Wirtschaftsforsch 78(2):122-145

Grabka MM, Frick JR (2003) Imputation of item-non-response on income questions in the SOEP 1984-2002. DIW research note 29, berlin. http://www.diw.de/documents/publikationen/73/diw_ 01.c.40797.de/diw_rn03-10-29.pdf. Zugegriffen: 5. Juni 2016

Groh-Samberg O (Hrsg) (2009) Armut, soziale Ausgrenzung und Klassenstruktur. Zur Integration multidimensionaler und längsschnittlicher Perspektiven. VS, Wiesbaden

Groves RM, Fowler FJ, Couper MP, Lepkowski JM, Singer E, Tourangeau R (2009) Survey methodology, 2. Aufl. Wiley, New York

King G (1995) Replication, replication. Polit Sci Polit 28(3):444-452

Kroh M (2010) Gewichtung im SOEP. Präsentation zum Workshop zur Nutzung des SOEP. http://www. diw.de/documents/dokumentenarchiv/17/diw_01.c.353319.de/soep_folien_gewichtung_2010.pdf. Zugegriffen: 20. Juli 2016

Kroh M (2012) Documentation of sample sizes and panel attrition in the German Socio Economic Panel (SOEP) (1984 until 2011). Data documentation 66, Berlin. http://www.diw.de/documents/ publikationen/73/diw_01.c.341747.de/diw_datadoc_2009-047.pdf. Zugegriffen: 15. Mai 2016

Krug G (2010) Fehlende Daten bei der Verknüpfung von Prozess- und Befragungsdaten. Ein empirischer Vergleich ausgewählter Missing Data Verfahren. Methoden, Daten, Analysen. Z Empir Sozialforsch 4(1):27-57

Leeuw ED, Collins M (1997) Data collection methods and survey quality: an overview. In: Lyberg LE, Biemer P, Collins M, Leeuw ED, Dippo C, Schwarz N, Trewin D (Hrsg) Survey measurement and process quality. Wiley, New York, S 199-220

Little RJA, Su H-L (1989) Item non-response in panel surveys. In: Kasprzyk D, Duncan G, Kalton G, Singh (Hrsg) Panel surveys. Wiley, New York, S 400-425

Schnell R, Gramlich T, Mosthaf A, Bender S (2010) Using complete administration data for nonresponse analysis: the PASS survey of low income households in Germany. Proceedings of Statistics Canada Symposium 2010. Social Statistics: The Interplay among Censuses, Surveys and Administrative Data. Statistics Canada, Ottawa, S 104-109

Schnell R, Hill PB, Esser E (2011) Methoden der empirischen Sozialforschung, 9. Aufl. Oldenbourg, München

Shikano S (2011) Einführung in die nichtparametrische Inferenz durch das Bootstrap-Verfahren. In: Best H, Wolf C (Hrsg) Handbuch der sozialwissenschaftlichen Datenanalyse. VS, Wiesbaden, S 191-204

Statistisches Bundesamt (2013) Einkommens- und Verbraucherstichprobe - Aufgabe, Methode und Durchführung der EVS. DeStatis Fachserie $15 \mathrm{Heft} 7$

Stauder J, Hüning W (2004) Die Messung von Äquivalenzeinkommen und Armutsquoten auf der Basis des Mikrozensus. In: Landesamt für Datenverarbeitung und Statistik Nordrhein-Westfalen (Hrsg) Statistische Analysen und Studien NRW, Band 13, Düsseldorf. http://www.mais.nrw.de/sozber/ sozialberichterstattung_nrw/grundlagen/stauder_huening_13_2004_1_.pdf. Zugegriffen: 20. Okt. 2016

Trappmann M, Beste J, Bethmann A, Müller G (2013) The PASS panel survey after six waves. J Labour Mark Res 46:275-281

Wagner GG, Frick JR, Schupp J (2007) The German Socio-Economic Panel Study (SOEP): evolution, scope and enhancements. Schmollers Jahrb Z Wirtsch Sozialwiss 127(1):139-169

Watson N, Starick R (2011) Evaluation of alternative income imputation methods for a longitudinal survey. J Off Stat 27(4):693-715 